\title{
Illumination and Color Control in Red-Green-Blue Light-Emitting Diode
}

\author{
Chun-Wen Tang, Bin-Juine Huang, and Shang-Ping Ying
}

\begin{abstract}
The concept of red-green-blue (RGB) light-emittingdiode (LED) lighting has gained wide attention during recent years and is now one of the targets for future lighting solutions. However, the self-heating of LEDs and environmental temperature variation lead to luminous intensity droop and lighting color drift. The main purpose of this research is to investigate a novel flux feedback and temperature feed-forward (FFB\&TFF) control structure for RGB LED lighting control system, in order to provide a wide color range of input commands and regulation of both luminous and color outputs. The work in this paper was carried out in three main steps. First, a thermal-electrical-luminous-chromatic model was derived and identified for RGB LED luminaire. Second, a converter and a compensator were derived and applied to input command conversion and temperature compensation, respectively. Finally, a diagonal proportional-integral controller designed by the decentralized control approach was implemented to regulate the lighting outputs of luminous intensity and chromaticity coordinates in CIE 1976 Uniform Chromaticity Space (UCS) diagram. The results of transient and steady-state experiments showed that the proposed control system was effective.
\end{abstract}

Index Terms-Decentralized control approach, flux feedback and temperature feed-forward (FFB\&TFF), red-green-blue (RGB) light-emitting-diodes (LEDs), system identification, thermalelectrical-luminous-chromatic model.

\section{INTRODUCTION}

$\mathbf{L}$ IGHT-EMITTING diodes (LEDs) are a highly effective lighting technology. Energy saving and long lifetimes are the main advantages in lighting applications. Red-green-blue (RGB) LED lighting is one of the most popular techniques for white light in the application of LCD backlighting, video projection and general lighting. Its chromatic changeability and wide color range can provide unique lighting experiences [1]. However, the luminous and color outputs are easily affected by self-heating of LEDs as well as environmental temperature variations [2], [3]. Thus, a control system for RGB LEDs is called for to regulate both luminous and color outputs. The key issue of effective regulation is a proper control structure design.

Manuscript received November 15, 2012; revised January 15, 2013; accepted February 20, 2013. Date of current version April 30, 2014. Recommended for publication by Associate Editor J. M. Alonso.

C.-W. Tang is with the Industrial Technology Research Institute, Hsinchu 31040, Taiwan (e-mail: CWTang@itri.org.tw).

B.-J. Huang is with the Mechanical Engineering Department, National Taiwan University, Taipei 106, Taiwan (e-mail: bjhuang@ seed.net.tw).

S.-P. Ying is with the Opto-Electronic System Engineering Department, Minghsin University of Science and Technology, Hsinchu 30401, Taiwan (e-mail: sbying@must.edu.tw).

Color versions of one or more of the figures in this paper are available online at http://ieeexplore.ieee.org.

Digital Object Identifier 10.1109/TPEL.2013.2251428
Several studies in the literature have proposed and compared to four control structures [4]-[10], that is, flux feedback (FFB), temperature feed-forward (TFF), FFB and temperature feedforward (FFB\&TFF), and color coordinates feedback (CCFB). Both FFB\&TFF and CCFB algorithms have shown better color stability. Because of easy implementation, CCFB methods have been studied by several authors [4]-[15] and shown effective color accuracy. However, the accuracy of color output would be restricted by temperature effect [7], inverted sensor matrix [6], [7], and spectra responses mismatch of the filtered color sensor [6], [8]. The FFB\&TFF method can avoid those drawbacks but require complex flux measuring technique [7]. Moreover, an algorithm of temperatures to color control commands instead of CCFB must be derived. Wang et al. [15] adopted a static lookup table as feed-forward compensator for FFB\&TFF method to implement the algorithm and input command conversion. However, the lookup table designed at 25 fixed operating conditions would confine the color range of the input commands. Therefore, the aim of this research is further to investigate a novel FFB\&TFF control structure that can apply a wide color range of input commands and achieve transient and steady-state requirements of both luminous and color outputs. The effect is experimentally verified.

The paper is organized as follows. In Section II, a novel FFB\&TFF control system with a thermal-electrical-luminouschromatic model of RGB LED luminaire, a converter, and a compensator is proposed. In Section III, the system identification of the RGB LED luminaire, the converter, and the compensator are presented. In Section IV, the controller design is further introduced. In Section V, the designed control system is implemented for performance analysis. Finally, some conclusions are drawn in Section VI.

\section{System DesCription AND MODELING}

\section{A. System Description}

In this study, a novel FFB\&TFF control structure of RGB LED lighting is proposed as shown in Fig. 1, in which $T_{\mathrm{CCr}}$ and $\Phi_{r}$ represent the correlated color temperature (CCT) command and total luminous intensity command, respectively, and $T_{\mathrm{CC}, \mathrm{OUT}}$ and $\Phi_{\mathrm{OUT}}$ represent CCT output and total luminous intensity output for lighting, respectively. A converter $N$ is applied to transform from $T_{\mathrm{CCr}}$ and $\Phi_{r}$ to three dependent signals: converted radiant power $L_{A}$, converted electrical power $P_{A}$, and converted junction temperature $T_{A}$. A compensator $M$ is applied to obtain the radiant compensation $L_{M}$ to compensate the $L_{A}$, by using the junction temperature of RGB LEDs $T_{\mathrm{LED}}$, the electrical power of RGB LEDs $P_{\mathrm{LED}}$, converted electrical 


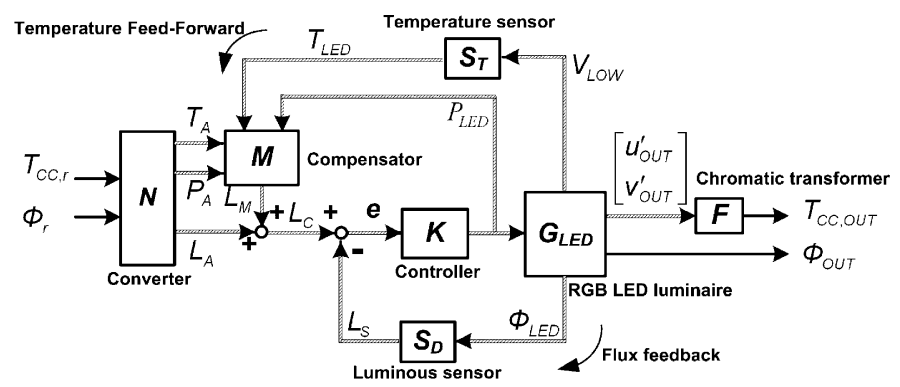

Fig. 1. Control structure of the RGB LED lighting control system (Solid lines: scalar signals; mesh lines: vector signals).

power $P_{A}$, and converted junction temperature $T_{A}$. The radiant command $L_{C}=L_{A}+L_{M}$ is a reference which leads $T_{\mathrm{CC} \text {, OUT }}$ and $\Phi_{\mathrm{OUT}}$ following $T_{\mathrm{CCr}}$ and $\Phi_{r}$, respectively. The controller $K$ is adopted to calculate a relevant $P_{\mathrm{LED}}$ according to the error signal $e$. The dynamics of RGB LED luminaire are modeled as $G_{\mathrm{LED}}$ with two outputs: $\Phi_{\mathrm{OUT}}$ as luminous output; and chromaticity coordinate in CIE1976 Uniform Chromaticity Space (UCS) diagram $\left[u_{\mathrm{OUT}}^{\prime}, v_{\mathrm{OUT}}^{\prime}\right]^{T}$ as color outputs. The $T_{\mathrm{CC}, \mathrm{OUT}}$ is transformed from $\left[u_{\mathrm{OUT}}^{\prime}, v_{\mathrm{OUT}}^{\prime}\right]^{T}$ by using a chromatic transformer $F$. The FFB loop carries out the radiant power of RGB LEDs $L_{S}$, acquired from the luminous intensity of RGB LEDs $\Phi_{\mathrm{LED}}$, tracking $L_{C}$ to regulate $\Phi_{\mathrm{OUT}}$; moreover, the TFF loop performs $T_{\mathrm{CC}, \mathrm{OUT}}$ following $T_{\mathrm{CCr}}$ since the $M$ provides accurate $L_{M}$. Furthermore, a proper controller of FFB loop can guarantee transient and steady-state performance of $\Phi_{\text {OUT }}$ and $T_{\mathrm{CC}, \mathrm{OUT}}$.

\section{B. Modeling of RGB LED Luminaire $G_{\mathrm{LED}}$}

RGB LED luminaire is a lighting fixture assembling multiple RGB LED lamps on a common heatsink. The RGB LED lamp is an electrical device, packaging red, green, and blue LED chips, driven by electrical powers for diverse color LEDs individually. According to the law of energy conservation, LEDs emit photons and simultaneously generate heat when electrical powers are applied. Photon emission will generate a spectrum, i.e., spectral power distribution (SPD), to produce luminous and color perception [16]. The heat will raise the junction temperature, which leads to low quantum efficiency [17], spectral shift of SPD [17], and current droop [18], [19], to cause color drift and luminous intensity droop.

In this study, the total luminous intensity output $\Phi_{\text {OUT }}$ and chromaticity coordinates outputs in CIE 1976 UCS diagram $\left[u_{\mathrm{OUT}}^{\prime}, v_{\mathrm{OUT}}^{\prime}\right]^{T}$ were both chosen as outputs of RGB LED luminaire as follows:

$$
\left[\begin{array}{c}
\tilde{\Phi}_{\mathrm{OUT}}(s) \\
\tilde{u}_{\mathrm{OUT}}^{\prime}(s) \\
\tilde{v}_{\mathrm{OUT}}^{\prime}(s)
\end{array}\right]=G_{\mathrm{LED}}(s) \cdot \tilde{P}_{\mathrm{LED}}(s)
$$

where $G_{\mathrm{LED}}$ is the RGB LED luminaire model, $P_{\mathrm{LED}}=\left[P_{R}\right.$ $\left.\begin{array}{ll}P_{G} & P_{B}\end{array}\right]^{T}$ is the electrical power of RGB LEDs, in which the subscripts " $R,{ },{ }_{G}$, " and " ${ }_{B}$, " respectively, represent the "red," "green," and "blue" components of signal; and " " represents perturbation. $G_{\mathrm{LED}}$ is a thermal-electrical-luminous-

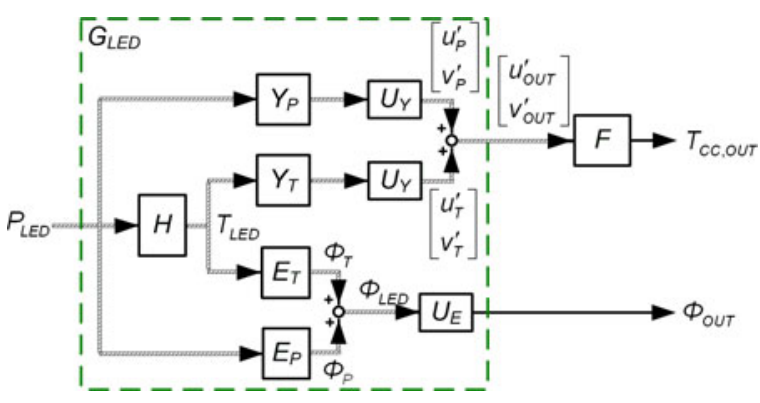

Fig. 2. Block diagram of thermal-electrical-luminous-chromatic model of RGB LED luminaire.

chromatic model, as shown in Fig. 2, as follows:

$$
G_{\mathrm{LED}}(s)=\left[\begin{array}{l}
U_{E} \cdot\left(E_{P}+E_{T} \cdot H(s)\right) \\
U_{Y} \cdot\left(Y_{P}+Y_{T} \cdot H(s)\right)
\end{array}\right]
$$

and can be divided into five perturbed submodels: electricalthermal (E-T) model $H$ represents thermal dynamics of luminaire; thermal-luminous (T-L) model $E_{T}$ and thermalchromatic (T-C) model $Y_{T}$ represent the opto-thermal effect; and electrical-luminous (E-L) model $E_{P}$ and electricalchromatic (E-C) model $Y_{P}$ represent the opto-electrical effect. $U_{E}=\left[\begin{array}{lll}1 & 1 & 1\end{array}\right]$ is the luminous summation unit, and $U_{Y}=$ $\left[\begin{array}{llllll}1 & 1 & 1 & 0 & 0 & 0 \\ 0 & 0 & 0 & 1 & 1 & 1\end{array}\right]$ is the chromatic summation unit. Referring to the derivation of [15] and [20]-[22], the total luminous intensity output is derived as follows:

$$
\begin{aligned}
\tilde{\Phi}_{\mathrm{OUT}}(s) & =U_{E} \cdot \tilde{\Phi}_{\mathrm{LED}}(s)=U_{E} \cdot\left(\tilde{\Phi}_{P}(s)+\tilde{\Phi}_{T}(s)\right) \\
& =U_{E} \cdot\left(E_{P} \cdot \tilde{P}_{\mathrm{LED}}(s)+E_{T} \cdot \tilde{T}_{\mathrm{LED}}(s)\right) \\
& =U_{E} \cdot\left(E_{P}+E_{T} \cdot H(s)\right) \cdot \tilde{P}_{\mathrm{LED}}(s) \\
& =U_{E} \cdot G_{E}(s) \cdot \tilde{P}_{\mathrm{LED}}(s)
\end{aligned}
$$

where $G_{E}$ is the luminous model, $\Phi_{\mathrm{LED}}=\left[\begin{array}{lll}\Phi_{R} & \Phi_{G} & \Phi_{B}\end{array}\right]^{T}$ is the luminous intensity of RGB LEDs, $T_{\mathrm{LED}}=\left[\begin{array}{lll}T_{R} & T_{G} & T_{B}\end{array}\right]^{T}$ is the junction temperature of RGB LEDs. All $H, E_{P}$, and $E_{T}$ are derived by the input-output relation as follows, respectively:

$$
\begin{aligned}
& H(s)=\left[\begin{array}{lll}
H_{R R}(s) & H_{G R}(s) & H_{B R}(s) \\
H_{R G}(s) & H_{G G}(s) & H_{B G}(s) \\
H_{R B}(s) & H_{G B}(s) & H_{B B}(s)
\end{array}\right] \\
& =\left[\begin{array}{lll}
\tilde{T}_{R}(s) / \tilde{P}_{R}(s) & \tilde{T}_{R}(s) / \tilde{P}_{G}(s) & \tilde{T}_{R}(s) / \tilde{P}_{B}(s) \\
\tilde{T}_{G}(s) / \tilde{P}_{R}(s) & \tilde{T}_{G}(s) / \tilde{P}_{G}(s) & \tilde{T}_{G}(s) / \tilde{P}_{B}(s) \\
\tilde{T}_{B}(s) / \tilde{P}_{R}(s) & \tilde{T}_{B}(s) / \tilde{P}_{G}(s) & \tilde{T}_{B}(s) / \tilde{P}_{B}(s)
\end{array}\right] \\
& \text { at } \tilde{T}_{a} \cong 0 \\
& E_{T}(s)=\left[\begin{array}{ccc}
E_{T R} & 0 & 0 \\
0 & E_{T G} & 0 \\
0 & 0 & E_{T B}
\end{array}\right] \\
& =\left[\begin{array}{ccc}
\tilde{\Phi}_{R}(s) / \tilde{T}_{R}(s) & 0 & 0 \\
0 & \tilde{\Phi}_{G}(s) / \tilde{T}_{G}(s) & 0 \\
0 & 0 & \tilde{\Phi}_{B}(s) / \tilde{T}_{B}(s)
\end{array}\right]
\end{aligned}
$$




$$
\begin{aligned}
E_{P}(s) & =\left[\begin{array}{ccc}
E_{P R} & 0 & 0 \\
0 & E_{P G} & 0 \\
0 & 0 & E_{P B}
\end{array}\right] \\
& =\left[\begin{array}{ccc}
\tilde{\Phi}_{R}(s) / \tilde{P}_{R}(s) & 0 & 0 \\
0 & \tilde{\Phi}_{G}(s) / \tilde{P}_{G}(s) & 0 \\
0 & 0 & \tilde{\Phi}_{B}(s) / \tilde{P}_{B}(s)
\end{array}\right] .
\end{aligned}
$$

Due to the same phenomenon of opto-electrical and optothermal effects, the dynamics of luminous responses can be extended to chromatic responses. The chromaticity coordinates outputs are similarly derived as follows:

$$
\begin{aligned}
{\left[\begin{array}{c}
\tilde{u}_{\mathrm{OUT}}^{\prime}(s) \\
\tilde{v}_{\mathrm{OUT}}^{\prime}(s)
\end{array}\right] } & =\left[\begin{array}{l}
\tilde{u}_{P}^{\prime}(s) \\
\tilde{v}_{P}^{\prime}(s)
\end{array}\right]+\left[\begin{array}{l}
\tilde{u}_{T}^{\prime}(s) \\
\tilde{v}_{T}^{\prime}(s)
\end{array}\right] \\
& =\left(U_{Y} \cdot Y_{P} \cdot \tilde{P}_{\mathrm{LED}}(s)+U_{Y} \cdot Y_{T} \cdot \tilde{T}_{\mathrm{LED}}(s)\right) \\
& =U_{Y} \cdot\left(Y_{P}+Y_{T} \cdot H(s)\right) \cdot \tilde{P}_{\mathrm{LED}}(s) \\
& =U_{Y} \cdot G_{Y}(s) \cdot \tilde{P}_{\mathrm{LED}}(s)
\end{aligned}
$$

where $G_{Y}$ is the chromatic model and the subscripts " $P$ " and " $T$ " represent the effects of "electrical power" and "junction temperature," respectively.

Both $Y_{P}$ and $Y_{T}$, are derived by the input-output relation as well. First, the T-C model $Y_{T}$ represents the influence on chromaticity coordinates of junction temperature as follows:

$$
\left[\begin{array}{c}
\tilde{u}_{T}^{\prime}(s) \\
\tilde{v}_{T}^{\prime}(s)
\end{array}\right]=U_{Y} \cdot Y_{T}(s) \cdot \tilde{T}_{\mathrm{LED}}(s) .
$$

Similar to the deviation of $E_{T}, Y_{T}$ is of zeroth order with a constant gain, defined in terms of linear perturbation concept as a $6 \times 3$ transfer function:

$$
\begin{aligned}
Y_{T} & =\left[\begin{array}{c}
Y_{T u} \\
Y_{T v}
\end{array}\right]=\left[\begin{array}{ccc}
Y_{T u R} & 0 & 0 \\
0 & Y_{T u G} & 0 \\
0 & 0 & Y_{T u B} \\
Y_{T v R} & 0 & 0 \\
0 & Y_{T v G} & 0 \\
0 & 0 & Y_{T v B}
\end{array}\right] \\
& =\left[\begin{array}{ccc}
\tilde{u}_{R}^{\prime}(s) / \tilde{T}_{R}(s) & 0 & 0 \\
0 & \tilde{u}_{G}^{\prime}(s) / \tilde{T}_{G}(s) & 0 \\
0 & 0 & \tilde{u}_{B}^{\prime}(s) / \tilde{T}_{B}(s) \\
\tilde{v}_{R}^{\prime}(s) / \tilde{T}_{R}(s) & 0 & 0 \\
0 & \tilde{v}_{G}^{\prime}(s) / \tilde{T}_{G}(s) & 0 \\
0 & 0 & \tilde{v}_{B}^{\prime}(s) / \tilde{T}_{B}(s)
\end{array}\right]
\end{aligned}
$$

where the $Y_{T}$ further divides into two diagonal $3 \times 3$ submodels $Y_{T u}$ and $Y_{T v}$ to represent the responses of the $u^{\prime}$-coordinate and $v^{\prime}$-coordinate, respectively. Second, the E-C model $Y_{P}$ represents the influence on chromaticity coordinates of electrical power as follows:

$$
\left[\begin{array}{c}
\tilde{u}_{P}^{\prime}(s) \\
\tilde{v}_{P}^{\prime}(s)
\end{array}\right]=U_{Y} \cdot Y_{P}(s) \cdot \tilde{P}_{\mathrm{LED}}(s)
$$

Similarly, $Y_{P}$ is of zeroth order with a constant gain, defined in terms of linear perturbation concept as a $6 \times 3$ transfer function:

$$
\begin{aligned}
Y_{P}= & {\left[\begin{array}{c}
Y_{P u} \\
Y_{P v}
\end{array}\right]=\left[\begin{array}{ccc}
Y_{P u R} & 0 & 0 \\
0 & Y_{P u G} & 0 \\
0 & 0 & Y_{P u B} \\
Y_{P v R} & 0 & 0 \\
0 & Y_{P v G} & 0 \\
0 & 0 & Y_{P v B}
\end{array}\right] } \\
= & {\left[\begin{array}{ccc}
\tilde{u}_{R}^{\prime}(s) / \tilde{P}_{R}(s) & 0 & 0 \\
0 & \tilde{u}_{G}^{\prime}(s) / \tilde{P}_{G}(s) & 0 \\
0 & 0 & \tilde{u}_{B}^{\prime}(s) / \tilde{P}_{B}(s) \\
\tilde{v}_{R}^{\prime}(s) / \tilde{P}_{R}(s) & 0 & 0 \\
0 & \tilde{v}_{G}^{\prime}(s) / \tilde{P}_{G}(s) & 0 \\
0 & 0 & \tilde{v}_{B}^{\prime}(s) / \tilde{P}_{B}(s)
\end{array}\right] }
\end{aligned}
$$

where the E-C model $Y_{P}$ divides into two $3 \times 3$ submodels $Y_{P u}$ and $Y_{P v}$, the same as in the $Y_{T}$.

In addition, the chromatic transformer $F$ represents a mapping from the chromaticity coordinate outputs $\left[u_{\mathrm{OUT}}^{\prime}, v_{\mathrm{OUT}}^{\prime}\right]^{T}$ onto CCT output $T_{\mathrm{CC}, \mathrm{OUT}}$. According to [23] and [24], CCT is obtained from a nonlinear approach as follows:

$$
F:\left\{\begin{array}{l}
T_{\mathrm{CC}, \mathrm{OUT}}=449 n^{3}+3525 n^{2}+6823.3 n+5520.3 \\
n=\frac{x-0.332}{0.1858-y} \\
x=\frac{9 u_{\text {OUT }}^{\prime}}{6 u_{\text {OUT }}^{\prime}-16 v_{\text {OUT }}^{\prime}+12} \\
y=\frac{4 v_{\text {OUT }}^{\prime}}{6 u_{\text {OUT }}^{\prime}-16 v_{\text {OUT }}^{\prime}+12}
\end{array}\right.
$$

where $n$ is the converting operator, and $x$ and $y$ are the chromaticity coordinates in CIE 1931 diagram.

\section{Modeling of Converter $N$}

Converter $N$ carries out a conversion to transform from the input commands of $T_{\mathrm{CC}, r}$ and $\Phi_{r}$, to three corresponding control signals: converted radiant power $L_{A}$, converted electrical power $P_{A}$, and converted junction temperature $T_{A}$; these five parameters were interdependent. The $N$ is described as follows:

$$
\left(L_{A}, T_{A}, P_{A}\right)=N\left(T_{\mathrm{CC}, r}, \Phi_{r}\right) .
$$

The $N$ must be consistent with the dynamics of RGB LED luminaire; therefore, $N$ is obtained from a set of experimental data of RGB LED luminaire, i.e., $T_{\mathrm{CC}, \mathrm{OUT}}, \Phi_{\mathrm{OUT}}, L_{S}, P_{\mathrm{LED}}$, and $T_{\mathrm{LED}}$, to construct the (13). Furthermore, $N$ could divide into three identical submodels when the set of experimental data 


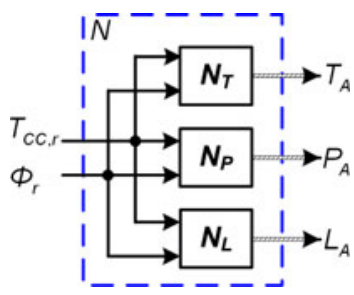

Fig. 3. Block diagram of converter $N$.

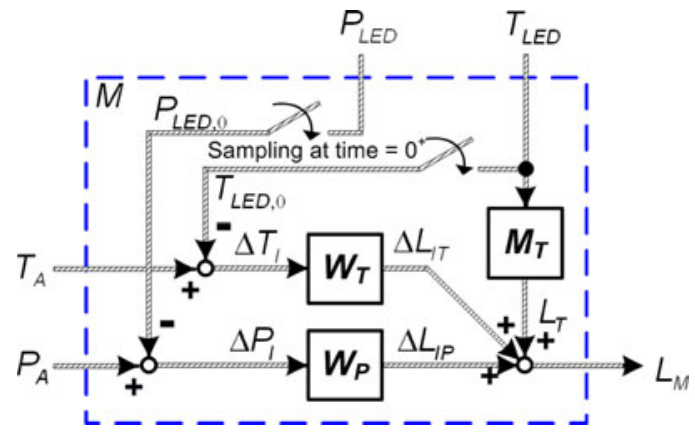

Fig. 4. Block diagram of compensator $M$.

is interdependent, described as follows:

$$
N:\left(\begin{array}{l}
L_{A}=N_{L}\left(T_{\mathrm{CC}, r}, \Phi_{r}\right) \\
T_{A}=N_{T}\left(T_{\mathrm{CC}, r}, \Phi_{r}\right) \\
P_{A}=N_{P}\left(T_{\mathrm{CC}, r}, \Phi_{r}\right)
\end{array}\right.
$$

where $N_{L}$ is the radiant converter, $N_{T}$ is the temperature converter, and $N_{P}$ is the electrical converter. Its detailed block diagram is shown in Fig. 3.

\section{Modeling of Compensator $M$}

$M$ is a feed-forward compensator, producing radiant compensation $L_{M}$, compensating the color drift and luminous intensity droop during the entire operating period due to junction temperature rise, and adjusting the signal mismatch between the converter $N$ outputs and initial responses of RGB LEDs for junction temperature and electrical power. $M$ consists of three submodels: temperature-compensator $M_{T}$, initial-temperaturecompensator $W_{T}$, and initial-electrical-compensator $W_{P}$, as shown in Fig. 4. The output of compensator $L_{M}$ is described as follows:

$$
\begin{aligned}
\tilde{L}_{M}(s) & =\tilde{L}_{T}(s)+\Delta L_{I T}+\Delta L_{I P} \\
& =M_{T}(s) \cdot \tilde{T}_{\mathrm{LED}}(s)+W_{T} \cdot \Delta T_{I}+W_{P} \cdot \Delta P_{I} .
\end{aligned}
$$

where $L_{T}$ is the feed-forward compensation, $\Delta L_{I T}$ the radiant difference of temperature, $\Delta L_{I P}$ the radiant difference of electrical power, $\Delta T_{I}$ the initial temperature difference defined the difference between converted junction temperature $T_{A}$, and initial junction temperature of RGB LEDs $T_{\mathrm{LED}, 0}$, i.e., $\Delta T_{I}=T_{A}-T_{\mathrm{LED}, 0}$. Similarly, the initial power difference is defined as $\Delta P_{I}=P_{A}-P_{\mathrm{LED}, 0}$.

The three submodels are derived by the input-output relation. First, temperature compensator $M_{T}$ represents the influence on feed-forward compensation $L_{T}$ of junction temperature $T_{\mathrm{LED}}$ as follows:

$$
\tilde{L}_{T}(s)=M_{T}(s) \cdot \tilde{T}_{\mathrm{LED}}(s)
$$

and is defined as a perturbed model as

$$
\begin{aligned}
M_{T}(s) & =\left[\begin{array}{lll}
N_{R R}(s) & N_{G R}(s) & N_{B R}(s) \\
N_{R G}(s) & N_{G G}(s) & N_{B G}(s) \\
N_{R B}(s) & N_{G B}(s) & N_{B B}(s)
\end{array}\right] \\
= & {\left[\begin{array}{lll}
\tilde{L}_{T R}(s) / \tilde{T}_{R}(s) & \tilde{L}_{T R}(s) / \tilde{T}_{G}(s) & \tilde{L}_{T R}(s) / \tilde{T}_{B}(s) \\
\tilde{L}_{T G}(s) / \tilde{T}_{R}(s) & \tilde{L}_{T G}(s) / \tilde{T}_{G}(s) & \tilde{L}_{T G}(s) / \tilde{T}_{B}(s) \\
\tilde{L}_{T B}(s) / \tilde{T}_{R}(s) & \tilde{L}_{T B}(s) / \tilde{T}_{G}(s) & \tilde{L}_{T B}(s) / \tilde{T}_{B}(s)
\end{array}\right] . }
\end{aligned}
$$

Second, initial-temperature-compensator $W_{T}$ and initialelectrical-compensator $W_{P}$ represent the radiant difference by initial temperature difference $\Delta T_{I}$ and initial electrical power difference $\Delta P_{I}$, respectively, as follows, respectively:

$$
\begin{aligned}
& \Delta L_{I T}=W_{T} \cdot \Delta T_{I} \\
& \Delta L_{I P}=W_{P} \cdot \Delta P_{I} .
\end{aligned}
$$

The $W_{T}$ and $W_{P}$ are defined as follows, respectively:

$$
\begin{aligned}
W_{T} & =\left[\begin{array}{lll}
W_{T R R} & W_{T G R} & W_{T B R} \\
W_{T R G} & W_{T G G} & W_{T B G} \\
W_{T R B} & W_{T G B} & W_{T B B}
\end{array}\right] \\
& =\left[\begin{array}{lll}
\Delta L_{I T R} / \Delta T_{I R} & \Delta L_{I T R} / \Delta T_{I G} & \Delta L_{I T R} / \Delta T_{I B} \\
\Delta L_{I T G} / \Delta T_{I R} & \Delta L_{I T G} / \Delta T_{I G} & \Delta L_{I T G} / \Delta T_{I B} \\
\Delta L_{I T B} / \Delta T_{I R} & \Delta L_{I T B} / \Delta T_{I G} & \Delta L_{I T B} / \Delta T_{I B}
\end{array}\right]
\end{aligned}
$$

$$
\begin{aligned}
W_{P} & =\left[\begin{array}{lll}
W_{P R R} & W_{P G R} & W_{P B R} \\
W_{P R G} & W_{P G G} & W_{P B G} \\
W_{P R B} & W_{P G B} & W_{P B B}
\end{array}\right] \\
= & {\left[\begin{array}{lll}
\Delta L_{I P R} / \Delta P_{I R} & \Delta L_{I P R} / \Delta P_{I G} & \Delta L_{I P R} / \Delta P_{I B} \\
\Delta L_{I P G} / \Delta P_{I R} & \Delta L_{I P G} / \Delta P_{I G} & \Delta L_{I P G} / \Delta P_{I B} \\
\Delta L_{I P B} / \Delta P_{I R} & \Delta L_{I P B} / \Delta P_{I G} & \Delta L_{I P B} / \Delta P_{I B}
\end{array}\right] . }
\end{aligned}
$$

The model of $M$ must be consistent with the dynamics of RGB LED luminaire; for the functions of $M$ is including the modification for $\Phi_{\mathrm{LED}}$ and $\left[u_{\mathrm{OUT}}^{\prime}, v_{\mathrm{OUT}}^{\prime}\right]^{T}$ due to junction temperature rise, and adjustment of signal mismatch between the outputs of converter $N$ and initial responses of RGB LEDs. Therefore, the $M$ can be derived from RGB LED luminaire models as mentioned previously, that is, $E_{P}, E_{T}, Y_{P}$, and $Y_{T}$. The detailed derivation is discussed in next section.

\section{SYSTEM IDENTIFICATION}

\section{A. Experimental Setup}

In this study, an RGB LED luminaire [21] was set up for system identification and lighting control; it is assembled by five RGB LED lamps [25] with a joint $900 \mathrm{~g}$ aluminum heatsink. 


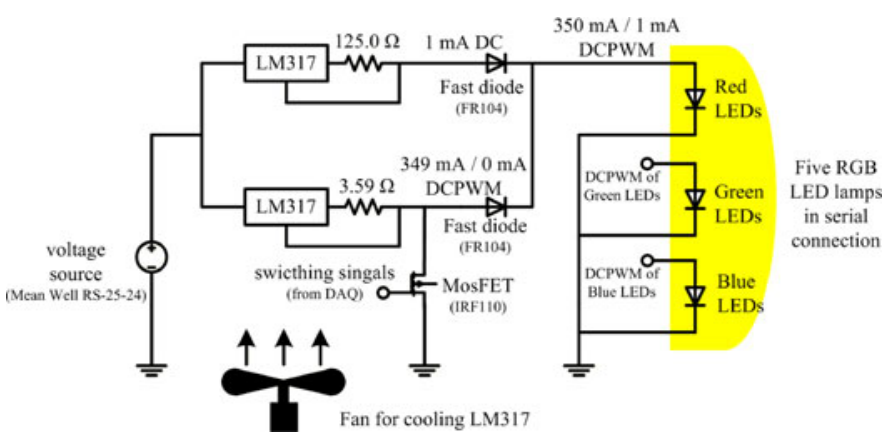

Fig. 5. Illustration of driver design for red LEDs.

TABLE I

ELECTRICAL POWER CONDITIONS OF RGB LED LUMINAIRE FOR WHITE LIGHT GENERATION

\begin{tabular}{|c|c|c|c|c|c|c|c|c|c|c|c|c|}
\hline CCT & \multicolumn{3}{|c|}{$3,500 \mathrm{~K}$} & \multicolumn{3}{c|}{$6,000 \mathrm{~K}$} & \multicolumn{3}{c|}{$8,000 \mathrm{~K}$} \\
\hline$\left(u^{\prime}, v^{\prime}\right)$ & \multicolumn{3}{|c|}{$(0.2346,0.5114)$} & \multicolumn{3}{c|}{$(0.2032,0.4708)$} & \multicolumn{3}{c|}{$(0.1951,0.4519)$} \\
\hline \multirow{3}{*}{$\begin{array}{c}\text { Electrical } \\
\text { power, }\end{array}$} & NO. & $P_{R}$ & $P_{G}$ & $P_{B}$ & NO. & $P_{R}$ & $P_{G}$ & $P_{B}$ & NO. & $P_{R}$ & $P_{G}$ & $P_{B}$ \\
\cline { 2 - 15 } & 89.0 & 25.7 & 12.0 & 4 & 87.0 & 32.0 & 27.0 & 7 & 85.4 & 33.2 & 35.0 \\
$P_{\text {LED }(\%)}$ & 27.5 & 22.1 & 11.5 & 5 & 71.0 & 26.2 & 22.0 & 8 & 68.8 & 26.9 & 28.0 \\
\cline { 2 - 13 } & 3 & 60.4 & 17.8 & 8.5 & 6 & 50.8 & 18.8 & 16.0 & 9 & 49.6 & 19.5 & 20.0 \\
\hline
\end{tabular}

Four lamps were installed in front of the luminaire for lighting and the fifth lamp with an Si photodiode [26] as the LED sensor was set inside the luminaire to measure junction temperature $T_{\mathrm{LED}}$ and radiant power $L_{S}$ of RGB LEDs.

Three color LEDs were independently driven by three drivers, as shown in Fig. 5, which were connected to identical color LEDs of five lamps in series connection. The three drivers independently produced direct current pulsewidth modulation (DCPWM) outputs. The ON/OFF current levels were supplied at $350 \mathrm{~mA} / 1 \mathrm{~mA}$, and the switching frequencies were fixed $120 \mathrm{~Hz}$ to avoid flicking perception [27]. The maximum electrical power in a single RGB LED lamp is $P_{\mathrm{LED}, \max }=[121$ $2.561 .27]^{T} \mathrm{~W} \equiv\left[\begin{array}{lll}100 & 100 & 100\end{array}\right]^{T} \%$, described in watt or percentage (\%), where the power levels are proportionally manipulated by duty cycle. The unit of electrical power is thus defined as "\%." The electrical power conditions of three CCTs, i.e., 3500, 6000, and $8000 \mathrm{~K}$, are listed in Table I for the following experiments. A thermal pad was attached to the luminaire to introduce extra thermal disturbance. By using the forward voltage method [28]-[30], the $T_{\mathrm{LED}}$ were estimated by measuring the average forward voltage of the LED sensor, $V_{\mathrm{LOW}}=$ $\left[\begin{array}{lll}V_{T R} & V_{T G} & V_{T B}\end{array}\right]^{T}$, while $1 \mathrm{~mA}$ constant current were applied during the OFF period of DCPWM, as follows:

$$
T_{\mathrm{LED}}=S_{T} \cdot V_{\mathrm{LOW}}
$$

where $S_{T}$ is the temperature sensitive parameter obtained from the calibration experiment by $1 \mathrm{~mA}$ constant current pulse with

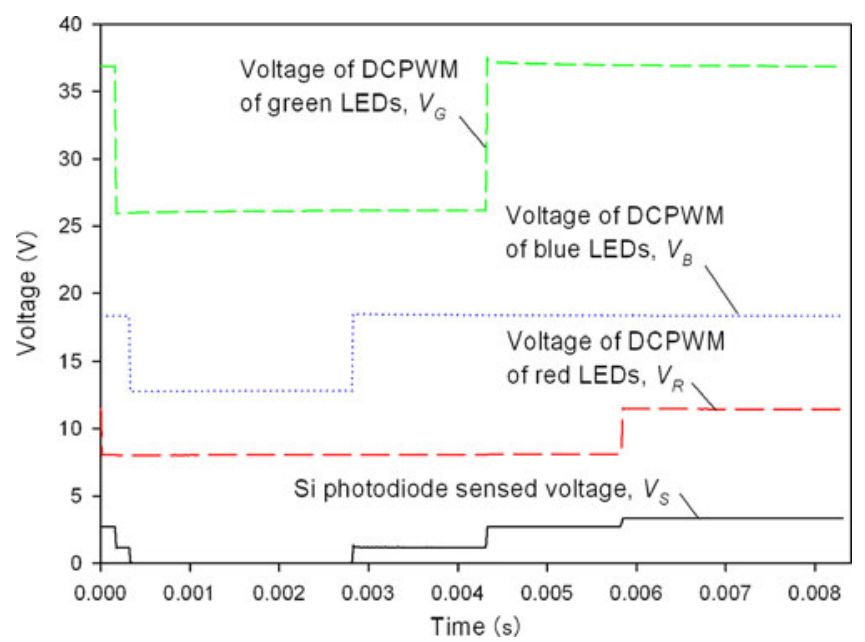

Fig. 6. Voltage responses of RGB LEDs and Si photodiode within one period.

$50 \mu$ s pulsewidth, as follows [15]:

$S_{T}=\left[\begin{array}{ccc}S_{T R} & 0 & 0 \\ 0 & S_{T G} & 0 \\ 0 & 0 & S_{T B}\end{array}\right]=\left[\begin{array}{ccc}1.82 & 0 & 0 \\ 0 & 5.90 & 0 \\ 0 & 0 & 2.20\end{array}\right] \times 10^{-3}$.

The luminous intensity of RGB LEDs $\Phi_{\mathrm{LED}}$ can be obtained by measuring the radiant powers $L_{S}=\left[\begin{array}{lll}L_{R} & L_{G} & L_{B}\end{array}\right]^{T}$ as follows:

$$
L_{S}=S_{D} \cdot \Phi_{\mathrm{LED}}
$$

The luminous detector model $S_{D}$ was then obtained by experimental calibration [15] as

$S_{D}=\left[\begin{array}{ccc}S_{D R} & 0 & 0 \\ 0 & S_{D G} & 0 \\ 0 & 0 & S_{D B}\end{array}\right]=\left[\begin{array}{ccc}0.0287 & 0 & 0 \\ 0 & 0.0212 & 0 \\ 0 & 0 & 0.1077\end{array}\right]$

Hence, the luminous intensity $\Phi_{\mathrm{LED}}$ can be measured directly and individually.

The radiant powers $L_{S}$ was measured by the LED sensor, i.e., the fifth lamp with an Si photodiode. The procedures were arranged as follows [4]. First, it is designed that the three DCPWM outputs were synchronous and their negative slope edge were occurred sequentially. The order was set red, green, and blue LEDs in turn, and the each time intervals of signals were set $2 \%$ duty cycle, i.e., $166.66 \mu$ s. The voltage responses of DCPWMs and Si photodiode are shown in Fig. 6. Second, the voltage falling edge of red LEDs was set as reference point to trigger the voltage measurement of $\mathrm{Si}$ photodiode at three specific time points, i.e., $1 \%, 3 \%$, and $99 \%$ duty cycle delayed to reference point. Its corresponding voltage values are equal to $V_{S, 1}=$ $V_{S G}+V_{S B}, V_{S, 3}=V_{S B}$, and $V_{S, 99}=V_{S R}+V_{S G}+V_{S B}$, respectively, where $V_{S R}, V_{S G}$, and $V_{S B}$ are Si photodiode sensed voltage of red, green, and blue LEDs. Third, the sensed voltages 
of RGB LEDs are decoupled as follows:

$$
\begin{aligned}
{\left[\begin{array}{c}
V_{S, 1} \\
V_{S, 3} \\
V_{S, 99}
\end{array}\right] } & =\left[\begin{array}{lll}
o & 1 & 1 \\
0 & 0 & 1 \\
1 & 1 & 1
\end{array}\right] \cdot\left[\begin{array}{c}
V_{S R} \\
V_{S G} \\
V_{S B}
\end{array}\right] \\
& {\left[\begin{array}{c}
V_{S R} \\
V_{S G} \\
V_{S B}
\end{array}\right]=\left[\begin{array}{lll}
o & 1 & 1 \\
0 & 0 & 1 \\
1 & 1 & 1
\end{array}\right]^{-1} \cdot\left[\begin{array}{c}
V_{S, 1} \\
V_{S, 3} \\
V_{S, 99}
\end{array}\right] } \\
& =\left[\begin{array}{c}
V_{S, 99}-V_{S, 1} \\
V_{S, 1}-V_{S, 3} \\
V_{S, 3}
\end{array}\right] .
\end{aligned}
$$

Finally, the radiant powers $L_{S}$ is obtained as follows:

$$
L_{S}=\left[\begin{array}{c}
L_{R} \\
L_{G} \\
L_{B}
\end{array}\right]=\left[\begin{array}{l}
D_{C R} \cdot V_{S R} \\
D_{C G} \cdot V_{S G} \\
D_{C B} \cdot V_{S B}
\end{array}\right]
$$

where $D_{C R}, D_{C G}$, and $D_{C B}$ are the duty cycles of red, green, and blue LEDs, respectively.

For identification, a set of testing apparatuses, the same as [15] (see Fig. 6), was installed to acquire total luminous intensity output $\Phi_{\mathrm{OUT}}$, chromaticity coordinate outputs $\left[u_{\mathrm{OUT}}^{\prime}, v_{\mathrm{OUT}}^{\prime}\right]^{T}$, and CCT output $T_{\mathrm{CC} \text {, OUT }}$. Moreover, the calculation of $\left[u_{\mathrm{OUT}}^{\prime}, v_{\mathrm{OUT}}^{\prime}\right]^{T}$ was obtained as follows [23]:

$$
\begin{array}{r}
{\left[\begin{array}{l}
u_{\mathrm{OUT}}^{\prime} \\
v_{\mathrm{OUT}}^{\prime}
\end{array}\right]=\left[\begin{array}{l}
4 X /(X+15 Y+3 Z) \\
9 Y /(X+15 Y+3 Z)
\end{array}\right]=C T(D),} \\
\text { where }\left\{\begin{array}{l}
X=\int_{380}^{780} D \cdot \bar{x} \cdot d \lambda \\
Y=\int_{380}^{780} D \cdot \bar{y} \cdot d \lambda \\
Z=\int_{380}^{780} D \cdot \bar{z} \cdot d \lambda
\end{array}\right.
\end{array}
$$

where $D$ is the SPD of RGB LEDs; $\bar{x}, \bar{y}$ and $\bar{z}$ are the color matching function; and $X, Y$ and $Z$ are the tristimulus values.

\section{B. Identification of $R G B$ LED Luminaire $G_{\mathrm{LED}}$}

The five submodels of $G_{\text {LED }}$ carried out system identification individually. First, the E-T model $H$ was obtained from (4) and is shown in [15], [20], and [21]. Second, the T-L model $E_{T}$ and the T-E model $E_{P}$ were obtained from (5) and (6), respectively, and the both models were identified as constant gain in [15] and [21]. Furthermore, the model of $E_{T}$ is observed as linearly electricaldependent (see Fig. 7); in other words, the $E_{T}$ is different from and linear to diverse electrical power conditions. In order to simplify the model description among operating range, $E_{T}$ can be characterized as follows:

$$
\begin{aligned}
E_{T} & =E_{T}\left(P_{\text {LED }}\right) \\
& =\left[\begin{array}{ccc}
-0.139 & 0 & 0 \\
0 & -0.151 & 0 \\
0 & 0 & -0.012
\end{array}\right] \cdot\left[\begin{array}{c}
P_{R} \\
P_{G} \\
P_{B}
\end{array}\right]
\end{aligned}
$$

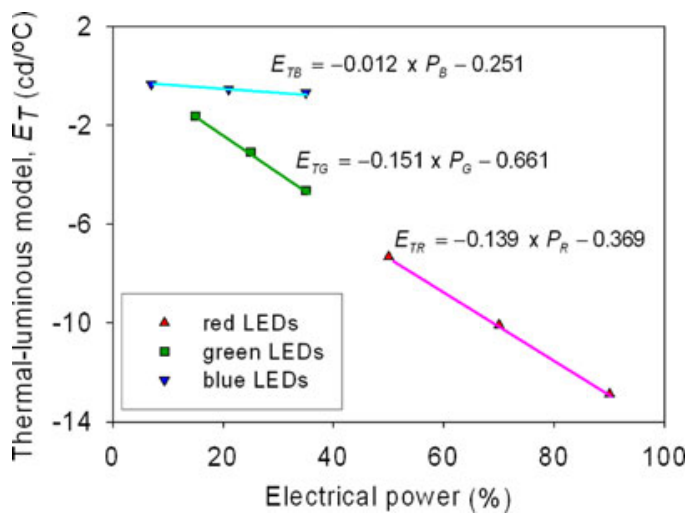

Fig. 7. Relations of thermal-luminous model $E_{T}$ and electrical power of RGB LEDs $P_{\mathrm{LED}}$.

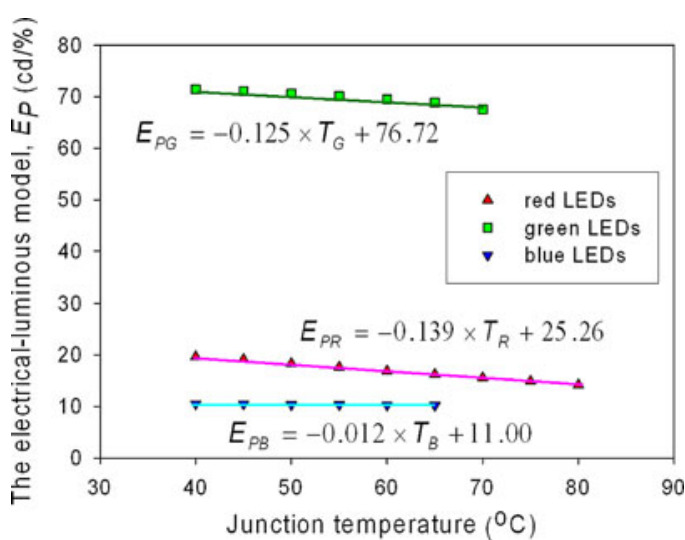

Fig. 8. Relations of electrical-luminous model $E_{P}$ and junction temperature of RGB LEDs $T_{\mathrm{LED}}$.

$$
+\left[\begin{array}{ccc}
-0.369 & 0 & 0 \\
0 & -0.661 & 0 \\
0 & 0 & -0.251
\end{array}\right] .
$$

Similarly, the model of $E_{P}$ was observed as linearly temperature-dependent (see Fig. 8) as well.

$$
\begin{aligned}
E_{P}= & E_{P}\left(T_{\mathrm{LED}}\right) \\
= & {\left[\begin{array}{ccc}
-0.139 & 0 & 0 \\
0 & -0.125 & 0 \\
0 & 0 & -0.012
\end{array}\right] \cdot\left[\begin{array}{c}
T_{R} \\
T_{G} \\
T_{B}
\end{array}\right] } \\
& +\left[\begin{array}{ccc}
25.26 & 0 & 0 \\
0 & 76.72 & 0 \\
0 & 0 & 11.00
\end{array}\right] .
\end{aligned}
$$

Third, the T-C model $Y_{T}$ was obtained from (9) and identified as a constant gain by linear regression. The $P_{\mathrm{LED}}$ were set constant, in turn, of the operating conditions given in Table I in the experiment. Accordingly, the $T_{\mathrm{LED}}$ was determined by self-heating and thermal interaction among three color LEDs. The $T_{\mathrm{LED}}$ rise would cause SPD variation of three color LEDs, respectively. In order to decouple the chromatic responses of the three color LEDs, the SPDs of RGB LEDs were thus defined as 


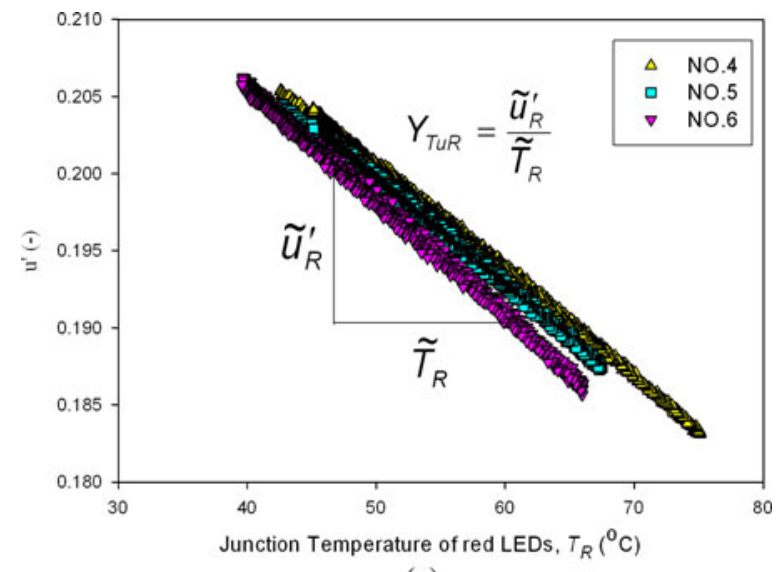

(a)

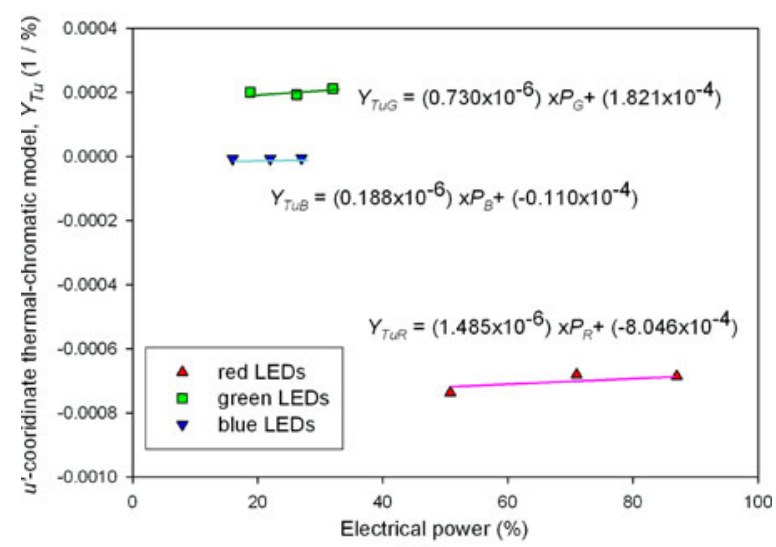

(b)

Fig. 9. $u^{\prime}$-coordinate experimental responses of thermal-chromatic model $Y_{T u}$ at CCT $6000 \mathrm{~K}$. (a) $u^{\prime}$-coordinate responses of red LEDs. (b) Relations of $Y_{T u}$ and $P_{\mathrm{LED}}$.

follows:

$$
\left\{\begin{array}{l}
\text { SPD of red LEDs: } D_{R}=\left.D\right|_{\lambda_{1}} ^{780 \mathrm{~nm}} \\
\text { SPD of green LEDs: } D_{G}=\left.D\right|_{\lambda_{2}} ^{\lambda_{1}} \\
\text { SPD of blue LEDs: } D_{B}=\left.D\right|_{380 \mathrm{~nm}} ^{\lambda_{2}}
\end{array}\right.
$$

where $\lambda_{1}$ is the wavelength of the spectrum trough between blue and green LEDs and $\lambda_{2}$ is the wavelength of the spectrum trough between green and red LEDs. Referring to (28), the perturbations of $\left[u_{T}^{\prime}, v_{T}^{\prime}\right]^{T}$ influenced by junction temperature of single-color LEDs were calculated from

$$
\left[\begin{array}{l}
\tilde{u}_{T}^{\prime} \\
\tilde{v}_{T}^{\prime}
\end{array}\right]=C T\left(\bar{D}+\tilde{D}_{i}\right)-C T(\bar{D}) \text {, for } \tilde{T}_{i} \text {, where } i=R, G, B \text {. }
$$

The modeling of $u^{\prime}$-coordinate T-C model for the red LEDs $Y_{\text {TuR }}$ at CCT $6000 \mathrm{~K}$ are shown in Fig. 9(a). Furthermore, $Y_{T}$ is observed as linearly electrical-dependent. In the example of CCT $6000 \mathrm{~K}$, the $u^{\prime}$-coordinate T-C model $Y_{T u}$ was depicted as a linear function of $P_{\text {LED }}$ [see Fig. 9(b)] as follows:

$$
\begin{gathered}
Y_{T}=Y_{T}\left(P_{\mathrm{LED}}\right)=\left[\begin{array}{ccc}
1.485 & 0 & 0 \\
0 & 0.730 & 0 \\
0 & 0 & 0.188 \\
0.495 & 0 & 0 \\
0 & -0.377 & 0 \\
0 & 0 & -1.009
\end{array}\right] \times 10^{-6} \cdot P_{\mathrm{LED}} \\
+\left[\begin{array}{ccc}
-8.046 & 0 & 0 \\
0 & 1.821 & 0 \\
0 & 0 & -0.110 \\
-1.947 & 0 & 0 \\
0 & -1.171 & 0 \\
0 & 0 & 2.981
\end{array}\right] \times 10^{-4} .
\end{gathered}
$$

Fourth, the E-C model $Y_{P}$ was obtained from (11) and identified as a constant gain by linear regression. The $P_{\text {LED }}$ were first set constant, in turn, of the operating conditions given in Table I in the experiment. Additionally, $P_{\mathrm{LED}}$ were applied, in turn, extra perturbations of $\tilde{P}_{R}= \pm 2.0 \%, \tilde{P}_{G}= \pm 1.5 \%$, and $\tilde{P}_{B}= \pm 1.0 \%$ with five divisions, individually. The extra perturbations are small enough to hold the chromaticity coordinates approximately remained at designing CCT. The perturbations of electrical powers were calculated from (28) as follows:

$$
\begin{aligned}
{\left[\begin{array}{l}
\tilde{u}_{P}^{\prime} \\
\tilde{v}_{P}^{\prime}
\end{array}\right]=} & {\left[\begin{array}{l}
\tilde{u}_{\text {OUT }}^{\prime} \\
\tilde{v}_{\text {OUT }}^{\prime}
\end{array}\right]-\left[\begin{array}{c}
\tilde{u}_{T}^{\prime} \\
\tilde{v}_{T}^{\prime}
\end{array}\right] } \\
= & \left(C T\left(\bar{D}+\tilde{D}_{i}\right)-C T(\bar{D})\right)-U_{Y} \cdot Y_{T} \cdot \tilde{T}_{\mathrm{LED}}, \\
& \text { for } \tilde{P}_{i}, \text { where } i=R, G, B .
\end{aligned}
$$

The modeling of $u^{\prime}$-coordinate E-C model for the red LEDs $Y_{P u R}$ at CCT $6000 \mathrm{~K}$ are shown in Fig. 10(a). Furthermore, $Y_{P}$ is observed as linearly electrical-dependent. In the example of CCT $6000 \mathrm{~K}$, the $u^{\prime}$-coordinate E-C model $Y_{P u}$ was depicted as a linear function of $P_{\text {LED }}$ [see Fig. 10(b)] as follows:

$$
\begin{aligned}
Y_{P}=Y_{P}\left(P_{\text {LED }}\right) & {\left[\begin{array}{ccc}
-0.259 & 0 & 0 \\
0 & 1.927 & 0 \\
0 & 0 & 0.326 \\
-0.054 & 0 & 0 \\
0 & -1.307 & 0 \\
0 & 0 & 2.245
\end{array}\right] \times 10^{-4} \cdot P_{\text {LED }} } \\
+ & {\left[\begin{array}{ccc}
3.271 & 0 & 0 \\
0 & -8.871 & 0 \\
0 & 0 & 1.235 \\
0.659 & 0 & 0 \\
0 & 5.962 & 0 \\
0 & 0 & -8.791
\end{array}\right] \times 10^{-3} }
\end{aligned}
$$




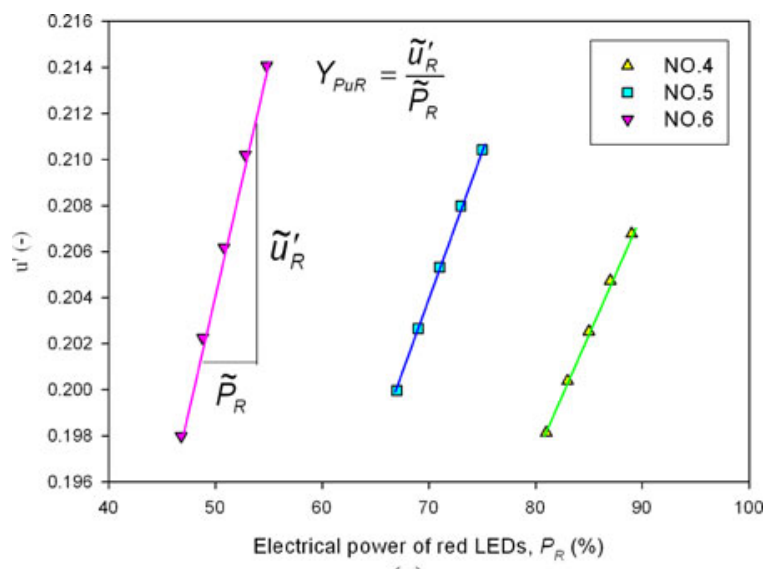

(a)

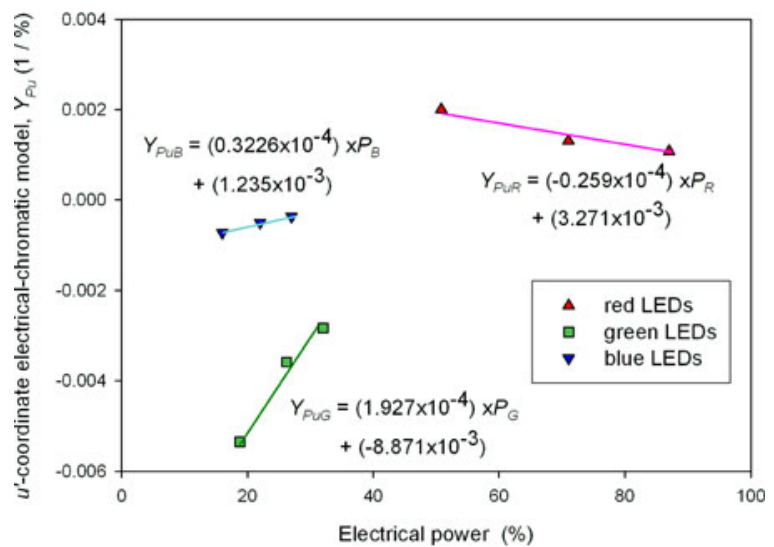

(b)

Fig. 10. $u^{\prime}$-coordinate experimental responses of electrical-chromatic model $Y_{P u}$ at CCT $6000 \mathrm{~K}$. (a) $u^{\prime}$-coordinate responses of red LEDs. (b) Relations of $Y_{P u}$ and $P_{\mathrm{LED}}$.

\section{Identification of Converter $N$}

The converter $N$ represents the transmission path from CCT command $T_{\mathrm{CCr}}$ and total luminous intensity command $\Phi_{r}$ to converted junction temperature $T_{A}$, converted electrical power $P_{A}$, and converted radiant power $L_{A}$. The three submodels $N_{L}, N_{P}$, and $N_{T}$ were carried out with curve-fitting technique, individually, by using the interdependent responses of the T-C model identification, i.e., $T_{\mathrm{CC}, \mathrm{OUT}}, \Phi_{\mathrm{OUT}}, T_{\mathrm{LED}}, P_{\mathrm{LED}}$, and $L_{S}$. In the example of $N_{L}, L_{A}$ can be obtained from the relations among $T_{\mathrm{CC}, \mathrm{OUT}}, \Phi_{\mathrm{OUT}}$, and $L_{S}$ according to (37). The identification consists of three steps: First, the $\Phi_{\mathrm{OUT}}$ of three CCTs are overlapping and can be fitted as a single linear function of the radiant power of green LED $L_{G}$, as shown in Fig. 11(a). Second, normalized radiant power $L_{N}$ is defined as follows:

$$
L_{N}=\left[\begin{array}{l}
L_{N R} \\
L_{N G} \\
L_{N B}
\end{array}\right]=\left[\begin{array}{l}
L_{R} /\left(L_{R}+L_{G}+L_{B}\right) \\
L_{G} /\left(L_{R}+L_{G}+L_{B}\right) \\
L_{B} /\left(L_{R}+L_{G}+L_{B}\right)
\end{array}\right] .
$$

The ratio of three elements of $L_{N}$ is constant to $\Phi_{\mathrm{OUT}}$, as depicted in Fig. 11(b). Third, the $T_{\mathrm{CC} \text {, OUT }}$ are linear to $L_{N}$, as shown in Fig. 11(c). Combining these three relations, $N_{L}$ is obtained as follows:

$N_{L}: L_{A} \equiv L_{S}=L_{N} \cdot \frac{L_{A G}}{L_{N G}}=\left[\begin{array}{c}L_{N R} \\ L_{N G} \\ L_{N B}\end{array}\right] \cdot \frac{L_{A G}}{L_{N G}}$,

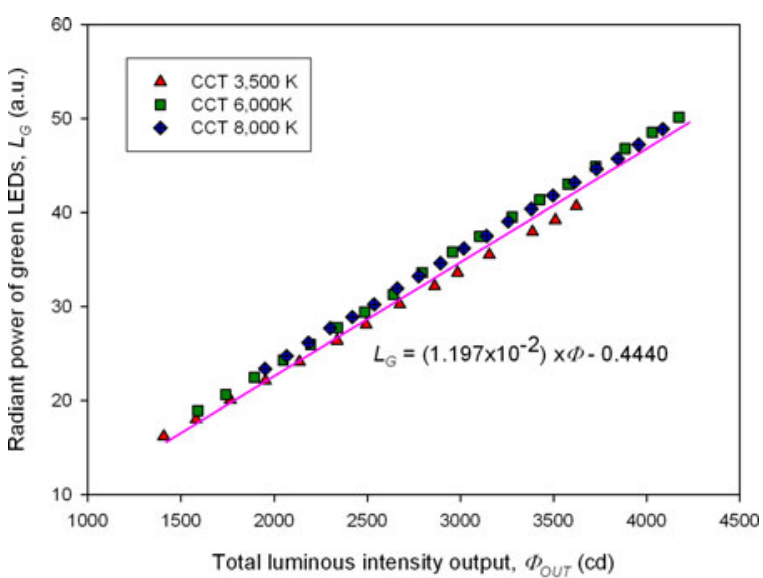

(a)

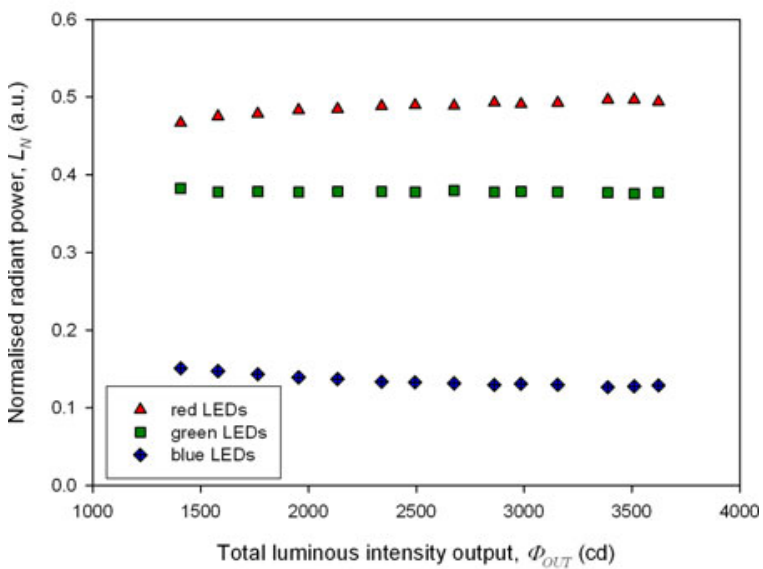

(b)

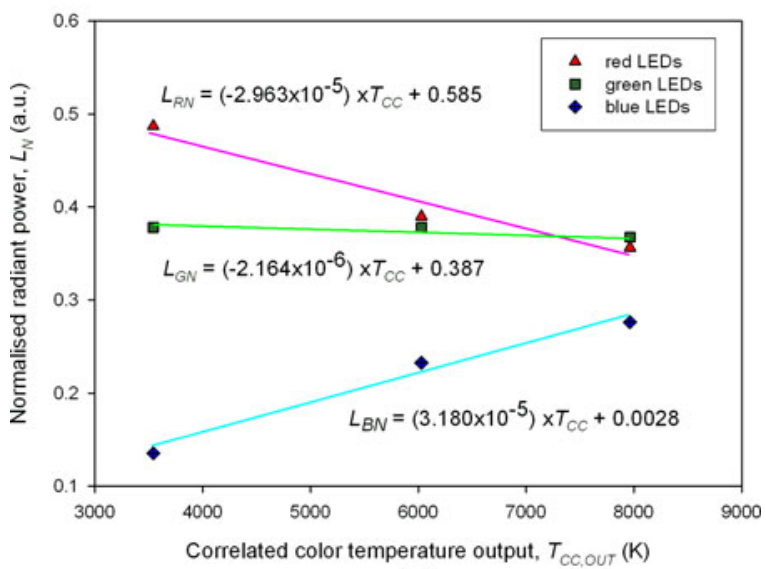

(c)

Fig. 11. Experimental responses for identification of radiant-converter $N_{L}$. (a) $\Phi_{\mathrm{OUT}}$ versus $L_{G}$. (b) $\Phi_{\mathrm{OUT}}$ versus $L_{N}$ at CCT $3500 \mathrm{~K}$. (c) $T_{\mathrm{CC}, \mathrm{OUT}}$ versus $L_{N}$.

$$
\text { where }\left\{\begin{array}{l}
L_{A G}=0.01197 \Phi_{r}-0.4440 \\
L_{N R}=-2.963 e^{-5} \cdot T_{\mathrm{CC}, r}+0.585 \\
L_{N G}=-2.164 e^{-6} \cdot T_{\mathrm{CC}, r}+0.387 \\
L_{N B}=3.180 e^{-5} \cdot T_{\mathrm{CC}, r}+0.0028
\end{array} .\right.
$$

Similarly, the same analytical technique was applied for identification of $N_{P}$ and $N_{T} . N_{P}$ and the $N_{T}$ are given by, 
respectively,

$$
\begin{gathered}
N_{P}: P_{A} \equiv P_{\mathrm{LED}}=P_{N} \cdot \frac{P_{A G}}{P_{N G}}=\left[\begin{array}{c}
P_{N R} \\
P_{N G} \\
P_{N B}
\end{array}\right] \cdot \frac{P_{A G}}{P_{N G}}, \\
\text { where }\left\{\begin{array}{l}
P_{A G}=7.678 e^{-3} \Phi_{r}-0.386 \\
P_{R N}=-3.174 e^{-5} \cdot T_{\mathrm{CC}, r}+0.801 \\
P_{G N}=3.459 e^{-6} \cdot T_{\mathrm{CC}, r}+0.195 \\
P_{B N}=2.828 e^{-5} \cdot T_{\mathrm{CC}, r}+0.0004
\end{array}\right. \\
N_{T}: T_{A} \equiv T_{\mathrm{LED}}=T_{N} \cdot \frac{T_{A G}}{T_{N G}}=\left[\begin{array}{l}
T_{N R} \\
T_{N G} \\
T_{N B}
\end{array}\right] \cdot \frac{T_{A G}}{T_{N G}} \\
\text { where }\left\{\begin{array}{l}
T_{A G}=2.580 e^{-3} \Phi_{r}+36.38 \\
T_{N R}=-1.346 e^{-6} \cdot T_{\mathrm{CC}, r}+0.350 \\
T_{N G}=-7.156 e^{-7} \cdot T_{\mathrm{CC}, r}+0.333 \\
T_{N B}=2.062 e^{-6} \cdot T_{\mathrm{CC}, r}+0.317
\end{array}\right.
\end{gathered}
$$

where $P_{N}$ is the normalized electrical power and $T_{N}$ is the normalized junction temperature.

\section{Derivation of Compensator $M$}

The compensator $M$ produced radiant compensation $L_{M}$ by using initial temperature difference $\Delta T_{I}$ and initial electrical power difference $\Delta P_{I}$; in addition, $M$ must be consistent with the dynamics of RGB LED luminaire to compensate the color drift and luminous intensity droop. Therefore, we constructed a combined relation of $T_{\mathrm{LED}}, P_{\mathrm{LED}}, L_{S}, \Phi_{\mathrm{OUT}}, u_{\mathrm{OUT}}^{\prime}$, and $v_{\mathrm{OUT}}^{\prime}$ in advance. The combined relation sets the difference of $\Phi_{\mathrm{OUT}}, u_{\mathrm{OUT}}^{\prime}$, and $v_{\mathrm{OUT}}^{\prime}$ equal to zero and then transforms into $M . M$ can produce radiant compensation $L_{M}$ to modify radiant command $L_{C}$ according to the dynamics of RGB LED luminaire. Eventually, a proper $L_{C}$ set as reference can guar- antee luminous and chromatic stability by using the FFB loop control, as shown in Fig. 1.

The combined relation is solved by using the identified models as mentioned previously, i.e., $E_{P}, E_{T}, Y_{P}$, and $Y_{T}$. First, combining (3) and (24), $\Phi_{\mathrm{LED}}$ is given by

$$
\tilde{\Phi}_{\mathrm{LED}}(s)=S_{D}^{-1} \cdot \tilde{L}_{S}(s)=E_{P} \cdot \tilde{P}_{\mathrm{LED}}(s)+E_{T} \cdot \tilde{T}_{\mathrm{LED}}(s)
$$

then

$$
\tilde{P}_{\mathrm{LED}}(s)=\left(E_{P}^{-1} \cdot S_{D}^{-1}\right) \cdot \tilde{L}_{S}(s)-\left(E_{P}^{-1} \cdot E_{T}\right) \cdot \tilde{T}_{\mathrm{LED}}(s) .
$$

We capture the $u^{\prime}$-coordinate and $v^{\prime}$-coordinate models of (9) and (11), i.e., $Y_{P u}, Y_{P v}, Y_{T u}$, and $Y_{T v}$. Their relations are reconstructed by (40) as follows:

$$
\begin{aligned}
\begin{aligned}
{\left[\begin{array}{c}
\tilde{u}_{R}^{\prime}(s) \\
\tilde{u}_{G}^{\prime}(s) \\
\tilde{u}_{B}^{\prime}(s)
\end{array}\right]=} & Y_{P u} \cdot \tilde{P}_{\mathrm{LED}}(s)+Y_{T u} \cdot \tilde{T}_{\mathrm{LED}}(s) \\
= & \left(Y_{P u} \cdot E_{P}^{-1} \cdot S_{D}^{-1}\right) \cdot \tilde{L}_{S}(s) \\
& +\left(Y_{T u}-Y_{P u} \cdot\left(E_{P}^{-1} \cdot E_{T}\right)\right) \cdot \tilde{T}_{\mathrm{LED}}(s) . \\
{\left[\begin{array}{c}
\tilde{v}_{R}^{\prime}(s) \\
\tilde{v}_{G}^{\prime}(s) \\
\tilde{v}_{B}^{\prime}(s)
\end{array}\right]=} & \left(Y_{P v} \cdot E_{P}^{-1} \cdot S_{D}^{-1}\right) \cdot \tilde{L}_{S}(s) \\
& +\left(Y_{T v}-Y_{P v} \cdot\left(E_{P}^{-1} \cdot E_{T}\right)\right) \cdot \tilde{T}_{\mathrm{LED}}(s) .
\end{aligned}
\end{aligned}
$$

Referring to the definition of (3) and (24), the total luminous intensity output $\Phi_{\mathrm{OUT}}$ is described in

$$
\tilde{\Phi}_{\mathrm{OUT}}=U_{E} \cdot \tilde{\Phi}_{\mathrm{LED}}(s)=U_{E} \cdot S_{D}^{-1} \cdot \tilde{L}_{S}(s)
$$

Combining (41)-(43) and the definition of $T_{\mathrm{LED}}, u_{\mathrm{OUT}}^{\prime}$, and $v_{\text {OUT }}^{\prime}$ into a $12 \times 12$ simultaneous equation yields the following, as shown $(*)$, at the bottom of this page.

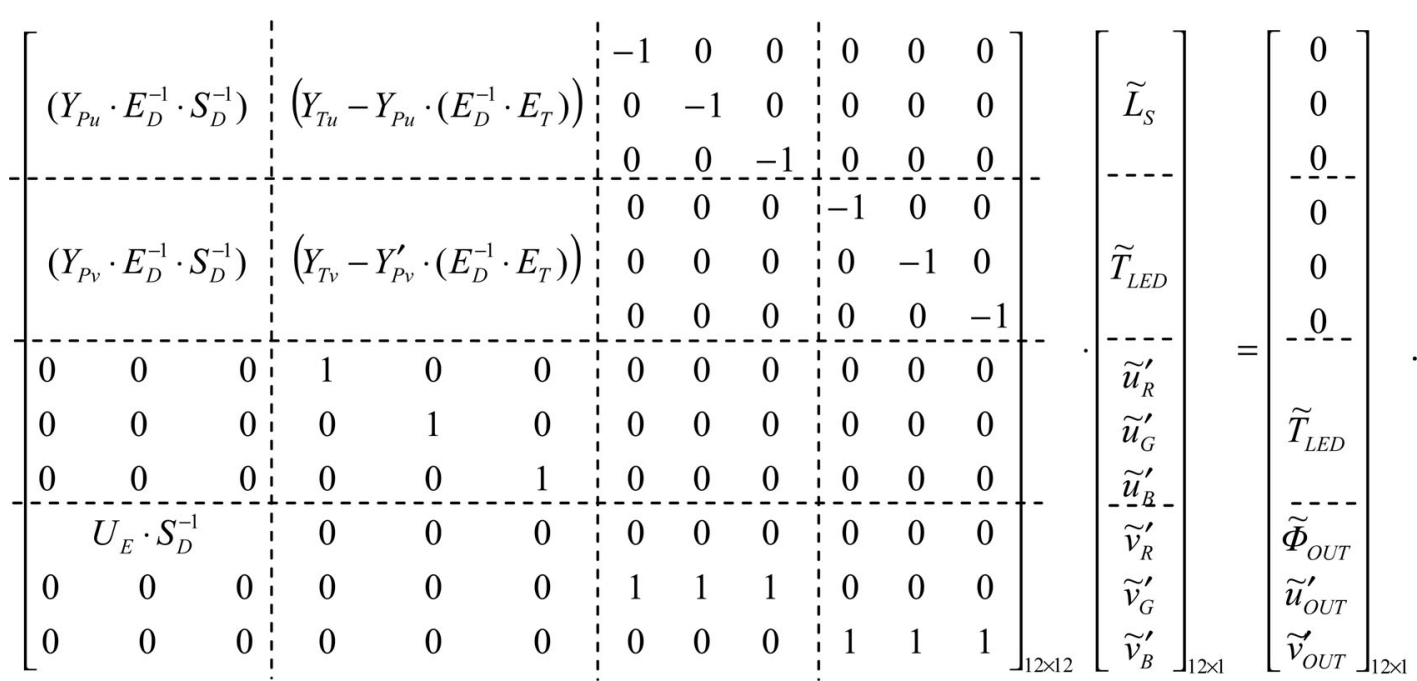


The solution is, as shown (44), at the bottom of the page.

Referring to (1), (3), and (7), the perturbation " " is redefined as difference " $\Delta$ " for the RGB LED luminaire outputs at the initial moment as follows:

$$
\left[\begin{array}{c}
\tilde{\Phi}_{\mathrm{OUT}} \\
\tilde{u}_{\mathrm{OUT}}^{\prime} \\
\tilde{v}_{\mathrm{OUT}}^{\prime}
\end{array}\right] \triangleq\left[\begin{array}{c}
\Delta \Phi_{I} \\
\Delta u_{I}^{\prime} \\
\Delta v_{I}^{\prime}
\end{array}\right]=\left[\begin{array}{c}
U_{E} \cdot E_{T} \\
U_{Y} \cdot Y_{T}
\end{array}\right] \cdot \Delta T_{I}+\left[\begin{array}{c}
U_{E} \cdot E_{P} \\
U_{Y} \cdot Y_{P}
\end{array}\right] \cdot \Delta P_{I} .
$$

Substituting (45) into the upper three rows of (44), we get

$$
\begin{aligned}
\tilde{L}_{S}= & {\left[\begin{array}{lll}
F_{7,1} & F_{8,1} & F_{9,1} \\
F_{7,2} & F_{8,2} & F_{9,2} \\
F_{7,3} & F_{8,3} & F_{9,3}
\end{array}\right] \cdot \tilde{T}_{\mathrm{LED}}+\left[\begin{array}{lll}
F_{10,1} & F_{11,1} & F_{12,1} \\
F_{10,2} & F_{11,2} & F_{12,2} \\
F_{10,3} & F_{11,3} & F_{12,3}
\end{array}\right] } \\
& \cdot\left[\begin{array}{l}
\tilde{\Phi}_{\mathrm{OUT}} \\
\tilde{u}_{\text {OUT }}^{\prime} \\
\tilde{v}_{\mathrm{OUT}}^{\prime}
\end{array}\right] \\
\equiv & {\left[\begin{array}{lll}
F_{7,1} & F_{8,1} & F_{9,1} \\
F_{7,2} & F_{8,2} & F_{9,2} \\
F_{7,3} & F_{8,3} & F_{9,3}
\end{array}\right] \cdot \tilde{T}_{\mathrm{LED}}+\left[\begin{array}{lll}
F_{10,1} & F_{11,1} & F_{12,1} \\
F_{10,2} & F_{11,2} & F_{12,2} \\
F_{10,3} & F_{11,3} & F_{12,3}
\end{array}\right] } \\
& \cdot\left(\left[\begin{array}{ll}
U_{E} \cdot E_{T} \\
U_{Y} \cdot Y_{T}
\end{array}\right] \cdot \Delta T_{I}+\left[\begin{array}{l}
U_{E} \cdot E_{P} \\
U_{Y} \cdot Y_{P}
\end{array}\right] \cdot \Delta P_{I}\right) .
\end{aligned}
$$

Referring to (15), the three sub-models of compensator, i.e., $M_{T}, W_{T}, W_{P}$, are modeled in comparison to (46) as follows:

$$
\begin{aligned}
M_{T}= & {\left[\begin{array}{lll}
F_{7,1} & F_{8,1} & F_{9,1} \\
F_{7,2} & F_{8,2} & F_{9,2} \\
F_{7,3} & F_{8,3} & F_{9,3}
\end{array}\right] } \\
W_{T}= & {\left[\begin{array}{lll}
F_{10,1} & F_{11,1} & F_{12,1} \\
F_{10,2} & F_{11,2} & F_{12,2} \\
F_{10,3} & F_{11,3} & F_{12,3}
\end{array}\right] \cdot\left[\begin{array}{l}
U_{E} \cdot E_{T} \\
U_{Y} \cdot Y_{T}
\end{array}\right] }
\end{aligned}
$$

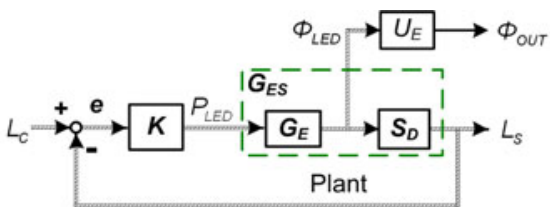

(a)

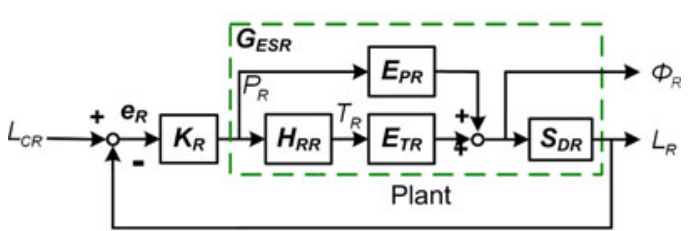

(b)

Fig. 12. Block diagram of FFB loops for controller design. (a) FFB loop. (b) Decoupled red LED loop (Solid lines: scalar signals; mesh lines: vector signals).

$$
W_{P}=\left[\begin{array}{lll}
F_{10,1} & F_{11,1} & F_{12,1} \\
F_{10,2} & F_{11,2} & F_{12,2} \\
F_{10,3} & F_{11,3} & F_{12,3}
\end{array}\right] \cdot\left[\begin{array}{l}
U_{E} \cdot E_{P} \\
U_{Y} \cdot Y_{P}
\end{array}\right] .
$$

Since $M$ is derived by $E_{P}, E_{T}, Y_{P}$, and $Y_{T}$, which are electricalor temperature-dependent, $M$ will constantly vary during the entire operating period. Therefore, the three submodels of $M$, i.e., $M_{T}, W_{T}$, and $W_{P}$, must be remodeled in each control period.

\section{Controller Design}

Regarding the FFB\&TFF structure shown in Fig. 1, it is noted that the performance of the RGB LED lighting control system is dominated mainly by the FFB loop if the converter $N$ and compensator $M$ provide exact radiant command $L_{C}$. Therefore, we segmented the FFB loop for the controller design, as depicted in Fig. 12(a). The plant of FFB loop $G_{E S}$ is defined as the average luminous model $G_{E 0}$ with luminous detector model $S_{D}$, i.e., $G_{E S}=S_{D} \cdot G_{E 0}$, where the $G_{E 0}$ is obtained by the

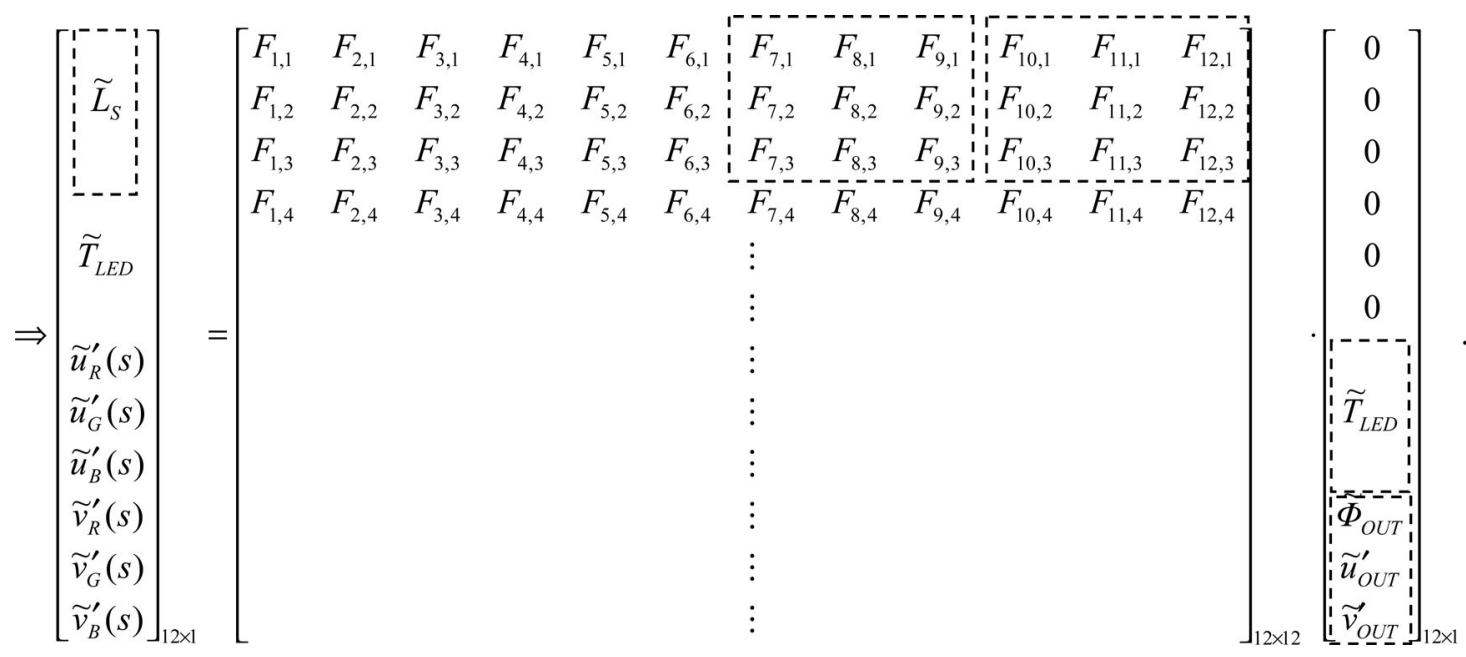


identification of $H, E_{P}$, and $E_{T}$.

$$
\begin{aligned}
G_{E 0}(s)= & {\left[\begin{array}{ccc}
G_{E 0, R R}(s) & G_{E 0, G R}(s) & G_{E 0, B R}(s) \\
G_{E 0, R G}(s) & G_{E 0, G G}(s) & G_{E 0, B G}(s) \\
G_{E 0, R B}(s) & G_{E 0, G B}(s) & G_{E 0, B B}(s)
\end{array}\right] } \\
= & {\left[\begin{array}{cc}
\frac{14.907 \cdot(s+0.00799)}{s+0.00083} & -\frac{0.582 \cdot(s+0.00368)}{s+0.00087} \\
-\frac{0.083 \cdot(s+0.00229)}{s+0.00083} & \frac{66.937 \cdot(s+0.00864)}{s+0.00087} \\
-\frac{0.014 \cdot(s+0.00212)}{s+0.00082} & -\frac{0.065 \cdot(s+0.00167)}{s+0.00083} \\
& -\frac{0.321 \cdot(s+0.00346)}{s+0.00085} \\
& -\frac{0.125 \cdot(s+0.00245)}{s+0.00084}
\end{array}\right] }
\end{aligned}
$$

In this study, the decentralized control approach was applied to simplification, that is, relative gain array (RGA) method [31], [32] was introduced to measure of the interactions and diagonal dominance. The RGA of the plant $G_{E S}$ is equal to identity matrix in the typical operating range $\omega=10^{-4}$ to $10^{3} \mathrm{rad} / \mathrm{s}$ as follows:

$$
\begin{aligned}
& \Lambda\left(G_{E S}\right) \\
& =G_{E S} \cdot *\left(G_{E S}^{T}\right)^{-1} \\
& =\left[\begin{array}{lll}
1.00+0.00 i & 0.00+0.00 i & 0.00+0.00 i \\
0.00+0.00 i & 1.00+0.00 i & 0.00+0.00 i \\
0.00+0.00 i & 0.00+0.00 i & 1.00+0.00 i
\end{array}\right] \equiv I \forall \omega
\end{aligned}
$$

where the operator “**" denotes Schur (element by element) matrix multiplication. According to the rule of pairing selection, the plant $G_{E S}$ is close to diagonal and essentially a collection of three independent subsystems. Therefore, the FFB loop is proper to treat as three single-input-single-output (SISO) loops, and the controller $K$ is designed in diagonal form in proportionalintegral (PI) strategy as follows:

$$
\begin{aligned}
K(s) & =\left[\begin{array}{ccc}
K_{R}(s) & 0 & 0 \\
0 & K_{G}(s) & 0 \\
0 & 0 & K_{B}(s)
\end{array}\right] \\
& =\left[\begin{array}{ccc}
K_{P R}+K_{I R} / s & 0 & 0 \\
0 & K_{P G}+K_{I G} / s & 0 \\
0 & 0 & K_{P B}+K_{I B} / s
\end{array}\right] .
\end{aligned}
$$

According to the stability theorem [31], suppose that the plant $G$ is stable, if RGA $\Lambda(G)=I \forall \omega$, the stability of each of individual loops implies stability of the entire system. Therefore, the stability of the FFB loop is determined by the stability of the diagonal loops of three color LEDs independently. By using the Routh-Hurwitz stability test, the stabilities of three loops were evaluated, respectively. The stability conditions were $K_{P R}>$

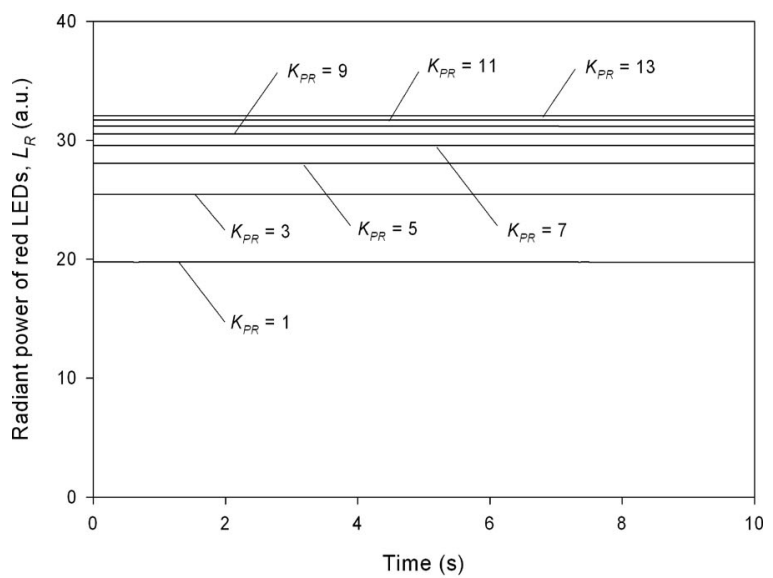

(a)

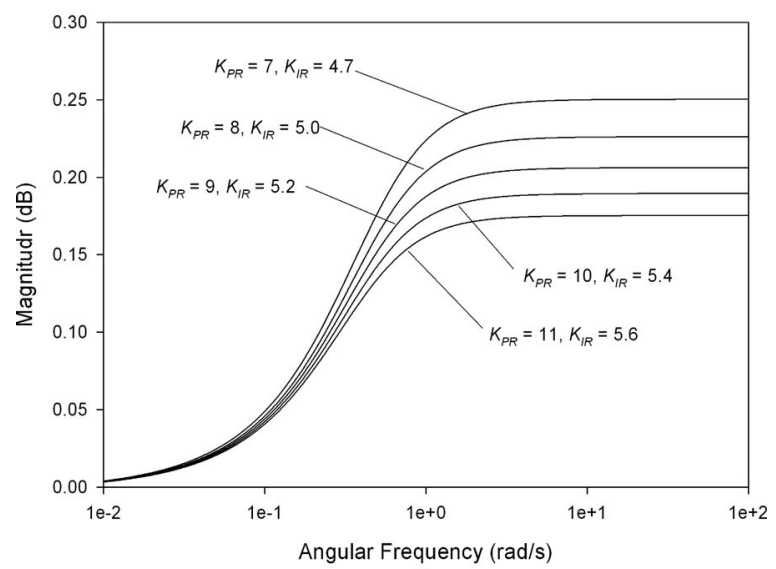

(b)

Fig. 13. Simulation results of decoupled red LED loop. (a) Time responses of proportional control test. (b) Frequency responses of sensitivity function $S_{E} R$ for PI control test.

-1.75 and $K_{I R}>0$ for red, $K_{P G}>-0.66$ and $K_{I G}>0$ for green, and $K_{P B}>-0.89$ and $K_{I B}>0$ for blue LED loops.

The performance of PI controller is tuned by using SIMULINK in MATLAB. In this study, the control system requirements are defined as follows:

1) rise time (time for step response from $10 \%$ to $90 \%$ ) $<1 \mathrm{~s}$;

2) settling time (time to reach $98 \%$ of reference) $<4$ s.

Using the time-domain simulation, optimal tuning may be carried out by finding the minimum of the integral of the absolute value of the error (IAE), which also satisfies the aforementioned system requirements, as follows:

$$
\mathrm{IAE}_{i}=\int_{0}^{\infty}\left|e_{i}(t)\right| d t, \quad \text { where } i=R, G, B
$$

where the error is $e=\left[\begin{array}{lll}e_{R} & e_{G} & e_{B}\end{array}\right]^{T}=\tilde{L}_{c}-\tilde{L}_{S}$.

The tuning procedures are simulated by step test. In the example of the decouple red LED loop [see Fig. 12(b)], the integral gain $K_{I R}$ is first set at zero in order to find the effective range of proportional gain $K_{P R}$. The simulated responses of $P$ control are not significant since the $K_{P R}>11.0$, as shown in Fig. 13 (a). Second, the $K_{P R}=7.0-11.0$ chosen for finding the minimum integral gain $K_{I R}$ satisfied the requirements of rising time and settling time. Its simulated results of IAE are listed in 
TABLE II

Simulation Results of PI Control Test of Decoupled FeEdBack Loops

\begin{tabular}{|c|c|c|c|c|c|c|c|c|}
\hline \multicolumn{3}{|c|}{ Red LEDs } & \multicolumn{3}{c|}{ Green LEDs } & \multicolumn{3}{c|}{ Blue LEDs } \\
\hline$K_{P R}$ & $K_{I R}$ & $I A E_{R}$ & $K_{P G}$ & $K_{I G}$ & $I A E_{G}$ & $K_{P B}$ & $K_{I B}$ & $I A E_{B}$ \\
\hline 7 & 4.7 & 11.040 & 5 & 2.5 & 9.234 & 3 & 2.3 & 12.225 \\
\hline 8 & 5.0 & 10.376 & 6 & 2.7 & 8.535 & 4 & 2.6 & 10.783 \\
\hline 9 & 5.2 & 9.976 & 7 & 2.8 & 8.214 & 5 & 2.8 & 9.986 \\
\hline 10 & 5.4 & 9.606 & 8 & 2.9 & 7.919 & 6 & 3.0 & 9.302 \\
\hline 11 & 5.6 & 9.262 & 9 & 3.0 & 7.646 & 7 & 3.0 & 8.708 \\
\hline
\end{tabular}

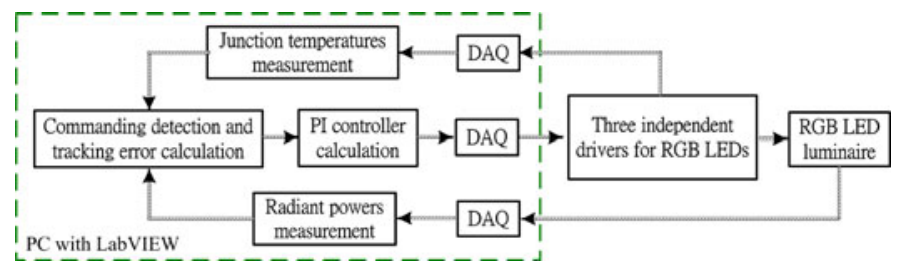

Fig. 14. Implementation of RGB LED lighting control system.

Table II. Third, the controller should be robust with respect to the plant uncertainty. A sensitivity function $S_{E R}$ is defined for the closed-loop transfer function $H_{E R}$ with respect to the variation of open-loop transfer function $G_{E S R}$ as

$S_{E R}(s)=\frac{\partial H_{E R}(s)}{\partial G_{E S R}(s)} \cdot \frac{G_{E S R}(s)}{H_{E R}(s)}=\frac{1}{1+K_{R}(s) \cdot G_{E S R}(s)}$.

The frequency responses of the $S_{E R}$ are plotted in the controller parameters of Table II, as shown in Fig. 13(b). Consequently, the acceptable PI controller, satisfying the system requirements, the stability condition, the criterion of minimum $\mathrm{IAE}_{R}$, and low sensitivity of the uncertainty of the plant $G_{E S R}$, is designed as $K_{P R}=11.0, K_{I R}=5.6$. Repeating the same procedures, the controller $K$ is finally designed as follows:

$$
K(s)=\left[\begin{array}{ccc}
11+5.6 / s & 0 & 0 \\
0 & 9+3.0 / s & 0 \\
0 & 0 & 7+3.0 / s
\end{array}\right] .
$$

\section{EXPERIMENTAL RESULT AND DISCUSSION}

A control system and a verification system, same as given in [15, Fig. 14 (b) and (c)], were set up for verification. For the control system, the designed converter $N$, compensator $M$, and controller $K$ were implemented by using a PC with LabVIEW and a data acquisition card (DAQ), as shown in Fig. 14. Because of the limitation of the DAQ, the sampling time of discrete control was set $T=0.1 \mathrm{~s}$. The discrete PI controller $K^{\prime}$ designed with zero-order hold was obtained as follows:

$$
K^{\prime}(z)=\frac{1}{z-1}\left[\begin{array}{ccc}
11 z+10.44 & 0 & 0 \\
0 & 9 z+8.7 & 0 \\
0 & 0 & 7 z+6.7
\end{array}\right] .
$$

For the verification system, the sampling time of data logging for total luminous intensity was $T=1.0 \mathrm{~s}$ due to the limitation of the General Purpose Interface Bus (GPIB) card.

Two experiments were designed to verify the system performances in total luminous intensity output $\Phi_{\mathrm{OUT}}$ and color difference of chromatic coordinate $\Delta u^{\prime} v^{\prime}=$ $\sqrt{\left(u_{\text {OUT }}^{\prime}-u_{0}^{\prime}\right)^{2}+\left(v_{\text {OUT }}^{\prime}-v_{0}^{\prime}\right)^{2}}$, where $u_{0}^{\prime}$ and $v_{0}^{\prime}$ are the initial desired chromaticity coordinates. In the transient experiments, the CCTs were set at constant 3800, 5000, and $6000 \mathrm{~K}$, respectively; and the total luminous intensity commands $\Phi_{r}$ were applied sequentially from 2000 to $3500 \mathrm{~cd}$ in intervals of $500 \mathrm{~cd}$ with $120 \mathrm{~s}$ for each step. The CCT conditions of 3800 and $5000 \mathrm{~K}$ differ from identification conditions 3500 and $6000 \mathrm{~K}$ in order to verify the control capability for various input commands. The experimental results are listed in Table III. Both rise time and settling time of $\Phi_{\mathrm{OUT}}$ are all within the requirements; the root-mean-square error (RMSE) of $\Phi_{\mathrm{OUT}}$ and their signalto-noise (SN) ratios show low steady-state error; and average $\Delta u^{\prime} v^{\prime}$ are shown low color drift as well. In the example of CCT $3800 \mathrm{~K}$, the experimental responses are revealed in Fig. 15. First, the $\Phi_{\mathrm{OUT}}$ follow the $\Phi_{r}$ and the $T_{\mathrm{CC}, \mathrm{OUT}}$ follow the $T_{\mathrm{CC}, \mathrm{r}}$, as shown in Fig. 15(a) and (b), respectively. Second, the color differences show effective regulation, as depicted in Fig. 15(b). Its responses at several step perturbation moments reveal large fluctuations; in that, the external electromagnetic disturbances, e.g., the power supply of driver, might interfere with the spectrometer; it is evident that there are no corresponding fluctuations to the $\Phi_{\mathrm{OUT}}, T_{\mathrm{LED}}$, and $L_{S}$. Third, the junction temperature $T_{\mathrm{LED}}$ and heatsink temperature $T_{b}$ revealed self-heating of LEDs, as shown in Fig. 15(c). Finally, measured radiant power $L_{S}$ and electrical power $P_{\mathrm{LED}}$ of RGB LEDs are depicted in Fig. 15(d) and (e), respectively.

In the steady-state experiments, the input commands were set $\Phi_{r}$ at constant $3000 \mathrm{~cd}$ and CCT at 3800,6000 , and $8000 \mathrm{~K}$, in turn, for the proposed control system. Moreover, the open-loop system driving constant electrical powers that are of the corresponding input commands at the initial time were tested as well. In addition, an extra $15 \mathrm{~W}$ thermal disturbance almost twice that of the power consumption of the RGB LED luminaire was applied during 4000-10000 s for both the systems. It caused an approximate $30^{\circ} \mathrm{C}$ junction temperature rise for each LEDs. The experimental results obtained during $t=9000-10000 \mathrm{~s}$ are listed in Table IV. For the proposed control system, the RMSE of $\Phi_{\text {OUT }}$ and average color difference $\Delta u^{\prime} v^{\prime}$ are both shown low steady-state error and low color drift, respectively. In the example of CCT $6000 \mathrm{~K}$, the experimental responses are shown in Fig. 16. All the $\Phi_{\mathrm{OUT}}, T_{\mathrm{CC}, \mathrm{OUT}}$, and $\Delta u^{\prime} v^{\prime}$ of the proposed control system are indicated well regulation, as shown in Fig. 16(a)-(c). Fig. 16(d) illustrates the temperature rise during the experiment. $T_{\mathrm{LED}}$ and $T_{b}$ dramatically rose at $4000 \mathrm{~s}$. 


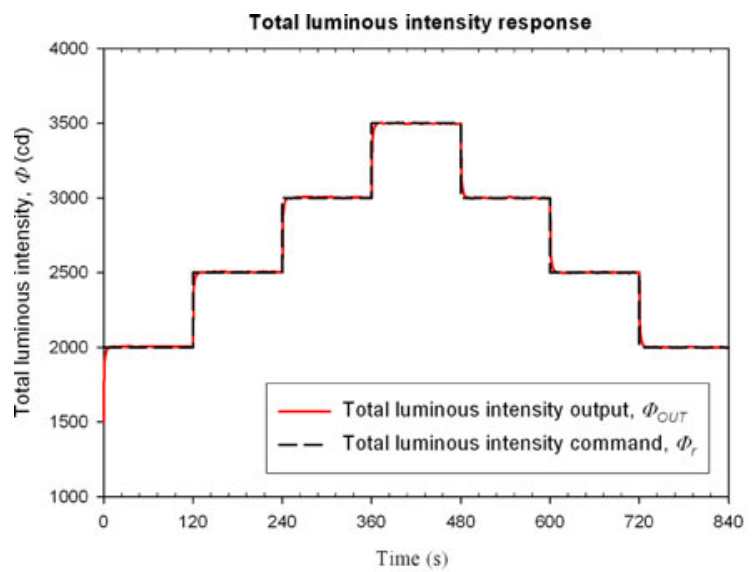

(a)

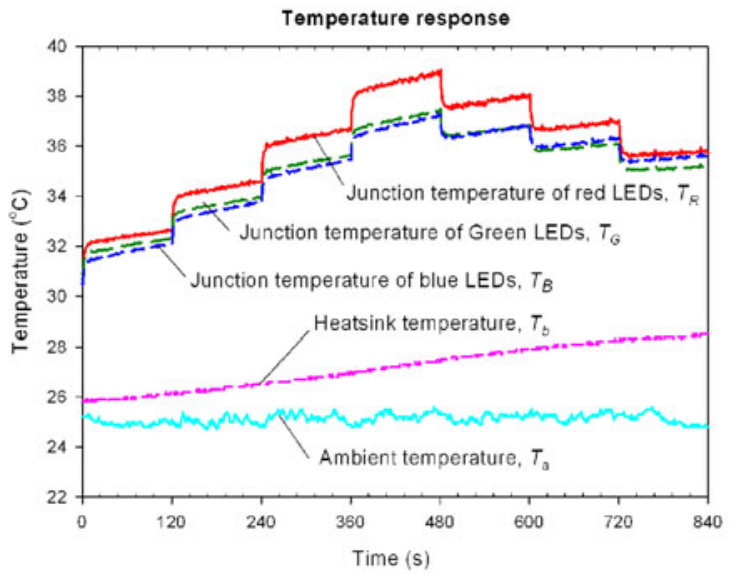

(c)

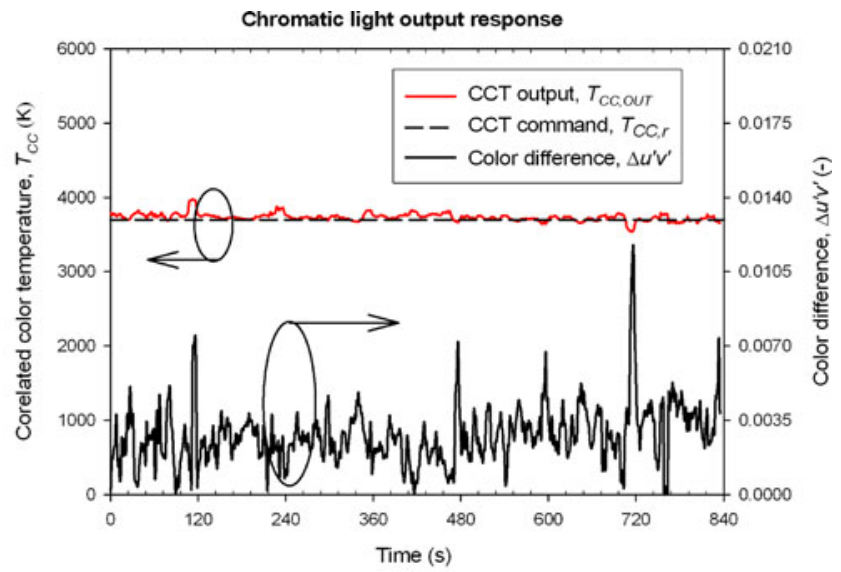

(b)

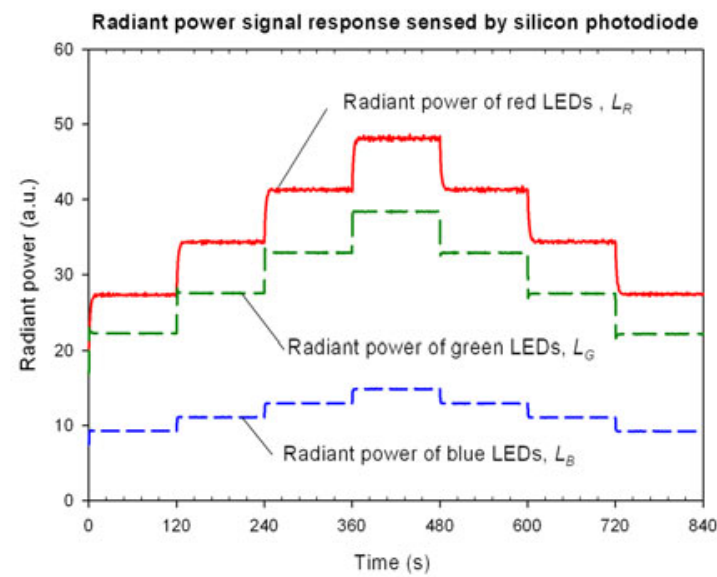

(d)

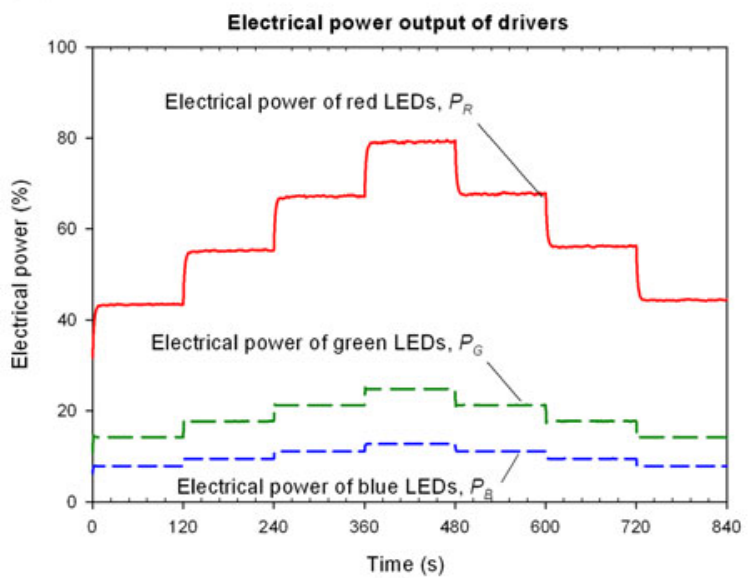

(e)

Fig. 15. Experimental responses to a serial total luminous intensity command and constant CCT $3800 \mathrm{~K}$ for proposed control system. (a) Total luminous intensity output. (b) Chromatic output. (c) Temperature. (d) Radiant power. (e) Electrical power.

Correspondingly, the $P_{\mathrm{LED}}$ showed noticeable compensation in Fig. 16(e).

These results show that the proposed control system, including the converter $N$, the compensator $M$, and the controller $K$, is effective; that is, our results indicate that both luminous and color outputs can achieve the requirements despite temperature rise and the system can operate at wide range color command. Regarding the results of Tables III and IV, the SN ratios of RMSE of $\Phi_{\mathrm{OUT}}$ could be treated as a steady-state error and all less than $2 \%$, where typically within $1 \%-0.1 \%$ for the transient experiment and $1.6 \%-0.16 \%$ for the steady-state experiment. Meanwhile, all of the $\Delta u^{\prime} v^{\prime}$ were typically within 0.0035 . These findings also support the hypothesis that the proposed control system can provide a wide color range of input command. It is evident that the system can operate at CCTs different from the identification conditions in the transient and steady-state experiments. Moreover, the RMSE of $\Phi_{\mathrm{OUT}}$, as well as $\Delta u^{\prime} v^{\prime}$, showed slight variation and inconsistency among the CCT or 


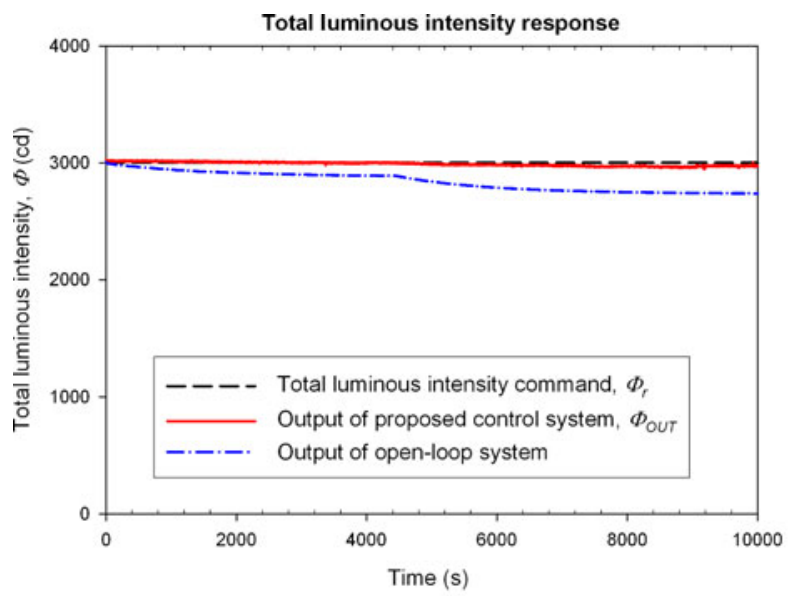

(a)

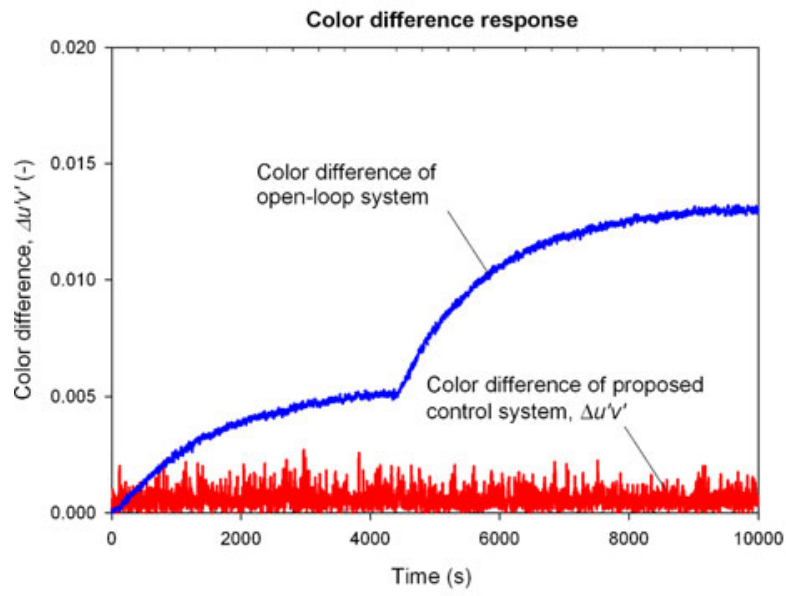

(c)

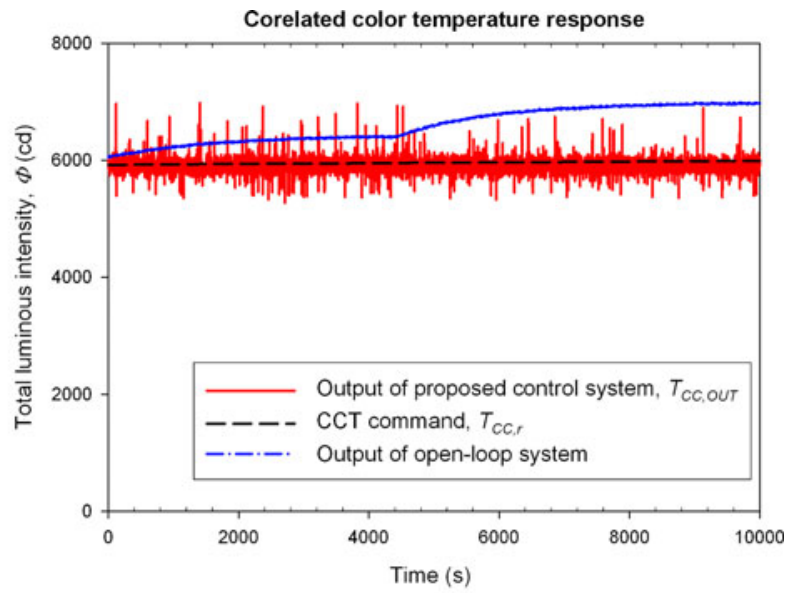

(b)

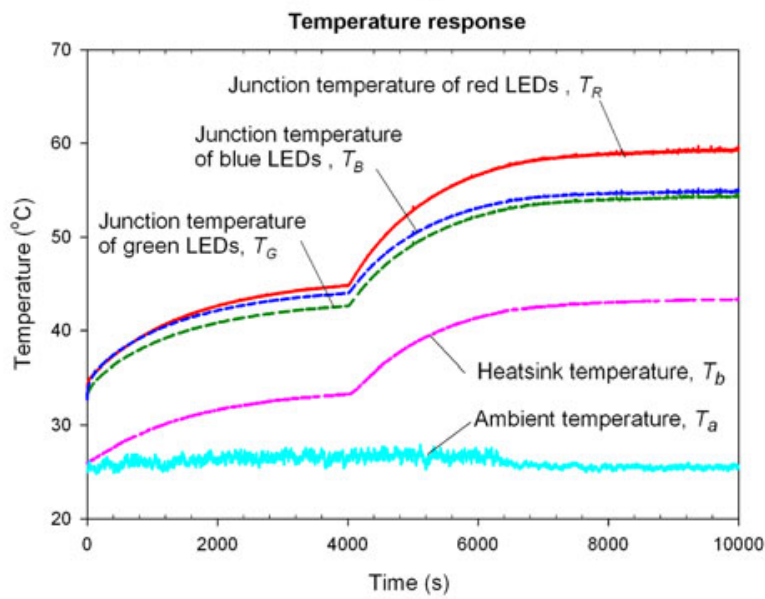

(d)

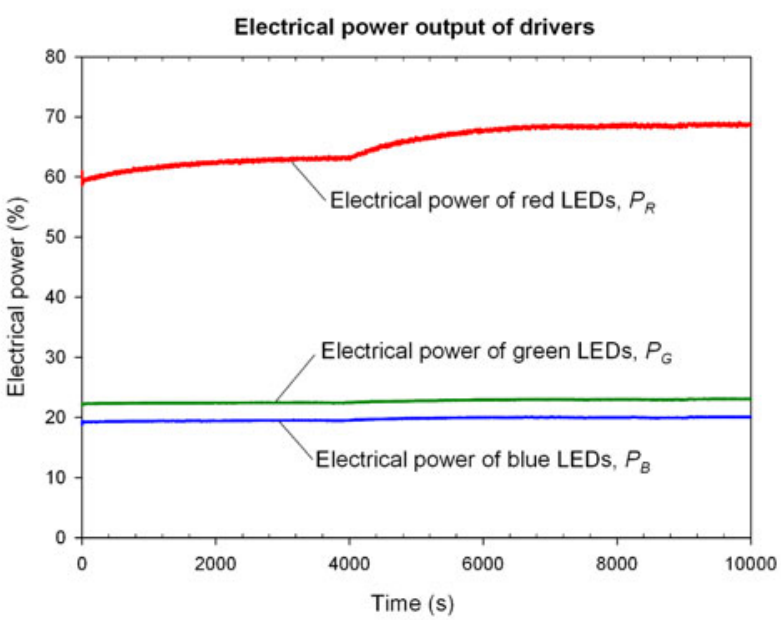

(e)

Fig. 16. Experimental responses to constant input command of $\Phi_{r}=3000 \mathrm{~cd}$ and $T_{\mathrm{CC}, r}=6000 \mathrm{~K}$ for open-loop and the proposed control system. (a) Total luminous intensity output. (b) CCT output. (c) Color difference output. (d) Temperature of proposed control system. (e) Electrical power of proposed control system.

electrical power conditions. For the transient experiment, the discordant results should be mainly caused by lack of performance of $K$ and accuracy of $N$; besides, it further introduces the lack of accuracy of $M$ for steady-state experiment due to large junction temperature rise. Therefore, the system performance could be further improved by obtaining precise $N$ and $M$ and more accurate controller design, e.g., Robust Control [15]. The accuracy of $N$ and $M$ can be improved by providing more experimental data of system identification for the four submodels, i.e., $E_{P}, E_{T}, Y_{P}$, and $Y_{T}$.

For the steady-state experiment, the $\Delta u^{\prime} v^{\prime}$ results showed 0.00297 (maximum) and 0.00171 (minimum) by approximate 
TABLE III

EXPERIMENTAL RESULTS OF THE TRANSIENT EXPERIMENTS

\begin{tabular}{|c|c|c|c|}
\hline & \multicolumn{3}{|c|}{ Correlated Color Temperature } \\
\hline & $3,800 \mathrm{~K}$ & $5,000 \mathrm{~K}$ & $6,000 \mathrm{~K}$ \\
\hline \multicolumn{4}{|l|}{ Total luminous intensity $\Phi_{O U T}$} \\
\hline \multicolumn{4}{|l|}{ rise time $T_{r}(\mathrm{~s})$} \\
\hline $2000 \mathrm{~cd} \rightarrow 2500 \mathrm{~cd}$ & 1.0 & 1.0 & 1.0 \\
\hline $2500 \mathrm{~cd} \rightarrow 3000 \mathrm{~cd}$ & 1.0 & 1.0 & 1.0 \\
\hline $3000 \mathrm{~cd} \rightarrow 3500 \mathrm{~cd}$ & 1.0 & 1.0 & 1.0 \\
\hline $3500 \mathrm{~cd} \rightarrow 3000 \mathrm{~cd}$ & 1.0 & 1.0 & 1.0 \\
\hline $3000 \mathrm{~cd} \rightarrow 2500 \mathrm{~cd}$ & 1.0 & 1.0 & 1.0 \\
\hline $2500 \mathrm{~cd} \rightarrow 2000 \mathrm{~cd}$ & 1.0 & 1.0 & 1.0 \\
\hline \multicolumn{4}{|l|}{ settling time $T_{s}(\mathrm{~s})$} \\
\hline $2000 \mathrm{~cd} \rightarrow 2500 \mathrm{~cd}$ & 3.0 & 3.0 & 3.0 \\
\hline $2500 \mathrm{~cd} \rightarrow 3000 \mathrm{~cd}$ & 3.0 & 3.0 & 3.0 \\
\hline $3000 \mathrm{~cd} \rightarrow 3500 \mathrm{~cd}$ & 3.0 & 3.0 & 3.0 \\
\hline $3500 \mathrm{~cd} \rightarrow 3000 \mathrm{~cd}$ & 3.0 & 1.0 & 4.0 \\
\hline $3000 \mathrm{~cd} \rightarrow 2500 \mathrm{~cd}$ & 4.0 & 1.0 & 4.0 \\
\hline $2500 \mathrm{~cd} \rightarrow 2000 \mathrm{~cd}$ & 4.0 & 3.0 & 3.0 \\
\hline \multicolumn{4}{|l|}{ RMSE (cd) } \\
\hline $2000 \mathrm{~cd}(60 \mathrm{~s}-120 \mathrm{~s}, 780 \mathrm{~s}-840 \mathrm{~s})$ & 3.50 & 20.68 & 8.31 \\
\hline $2500 \mathrm{~cd}(180 \mathrm{~s}-240 \mathrm{~s}, 660 \mathrm{~s}-720 \mathrm{~s})$ & 3.07 & 22.27 & 14.68 \\
\hline $3000 \mathrm{~cd}(300 \mathrm{~s}-360 \mathrm{~s}, 540 \mathrm{~s}-600 \mathrm{~s})$ & 3.39 & 20.69 & 21.70 \\
\hline $3500 \mathrm{~cd}(420 \mathrm{~s}-480 \mathrm{~s})$ & 3.94 & 20.02 & 27.96 \\
\hline \multicolumn{4}{|l|}{ SN ratio of RMSE (\%) } \\
\hline $2000 \mathrm{~cd}(60 \mathrm{~s}-120 \mathrm{~s}, 780 \mathrm{~s}-840 \mathrm{~s})$ & 0.18 & 1.03 & 0.42 \\
\hline $2500 \mathrm{~cd}(180 \mathrm{~s}-240 \mathrm{~s}, 660 \mathrm{~s}-720 \mathrm{~s})$ & 0.12 & 0.89 & 0.59 \\
\hline $3000 \mathrm{~cd}(300 \mathrm{~s}-360 \mathrm{~s}, 540 \mathrm{~s}-600 \mathrm{~s})$ & 0.11 & 0.69 & 0.72 \\
\hline $3500 \mathrm{~cd}(420 \mathrm{~s}-480 \mathrm{~s})$ & 0.11 & 0.57 & 0.80 \\
\hline \multicolumn{4}{|l|}{ Average color difference, $\Delta u^{\prime} v^{\prime}(-)$} \\
\hline $2000 \mathrm{~cd}(60 \mathrm{~s}-120 \mathrm{~s}, 780 \mathrm{~s}-840 \mathrm{~s})$ & 0.00313 & 0.00345 & 0.00225 \\
\hline $2500 \mathrm{~cd}(180 \mathrm{~s}-240 \mathrm{~s}, 660 \mathrm{~s}-720 \mathrm{~s})$ & 0.00269 & 0.00225 & 0.00142 \\
\hline $3000 \mathrm{~cd}(300 \mathrm{~s}-360 \mathrm{~s}, 540 \mathrm{~s}-600 \mathrm{~s})$ & 0.00287 & 0.00337 & 0.00295 \\
\hline $3500 \mathrm{~cd}(420 \mathrm{~s}-480 \mathrm{~s})$ & 0.00172 & 0.00340 & 0.00351 \\
\hline
\end{tabular}

$40{ }^{\circ} \mathrm{C}$ junction temperature variation. Earlier publications showed several comparable results for RGB LEDs as follows. Muthu et al. [4], [8] and Muthu and Gaines [9] reported that the
TABLE IV

EXPERIMENTAL RESULTS OF THE STEADY-STATE EXPERIMENTS

\begin{tabular}{|c|c|c|c|}
\hline & \multicolumn{3}{|c|}{ Correlated Color Temperature } \\
\hline & $3,800 \mathrm{~K}$ & $6,000 \mathrm{~K}$ & $8,000 \mathrm{~K}$ \\
\hline Total luminous intensity output $\Phi_{\text {OVT }}$ & & & \\
\hline RMSE (cd) & & & \\
\hline proposed control system & 47.91 & 27.23 & 4.75 \\
\hline open-loop system & 281.99 & 259.60 & 256.20 \\
\hline SN ratio of RMSE (\%) & & & \\
\hline proposed control system & 1.60 & 0.91 & 0.16 \\
\hline open-loop system & 9.40 & 8.65 & 8.54 \\
\hline Average color difference $\Delta u^{\prime} v^{\prime}(-)$ & & & \\
\hline proposed control system & 0.00297 & 0.00207 & 0.00171 \\
\hline open-loop system & 0.01292 & 0.01293 & 0.01268 \\
\hline
\end{tabular}

color point changes in CIE 1960 are $\Delta u v=0.004$ (FFB\&TFF), 0.006 (CCFB), and 0.008 (CCFB) for a heatsink temperature change of $50^{\circ} \mathrm{C}$. Hoelen et al. [5], [7] demonstrated a maximum color point error $\Delta u^{\prime} v^{\prime}$ in white of 0.007 (FFB\&TFF) with $35^{\circ} \mathrm{C}$ and 0.0092 (FFB\&TFF) and 0.0084 (CCFB) with $75^{\circ} \mathrm{C}$ heatsink temperature change, respectively. Deurenberg et al. [6] pointed out that the temperature induced color accuracy errors of average $\Delta u^{\prime} v^{\prime}=0.0035$ and 0.0048 , respectively, for the FFB \& TFF and CCFB systems for a heatsink temperature variation of $50^{\circ} \mathrm{C}$. Wang et al. [15] showed a typical $\Delta u^{\prime} v^{\prime}=$ 0.001 under $25^{\circ} \mathrm{C}$ junction temperature rise.

The proposed control system was discussed in-depth as follows. It appears that both $N$ and $M$ of the TFF loop provide accurate radiant command $L_{C}$ to the following FFB loop. The FFB loop implemented in three SISO loops regulates the luminous intensity of RGB LEDs $\Phi_{\mathrm{LED}}$ as well as the total luminous intensity output $\Phi_{\mathrm{OUT}}$. Moreover, the TFF loop compensates temperature variation further to regulate the color difference $\Delta u^{\prime} v^{\prime}$ and CCT output $T_{\mathrm{CC}, \mathrm{OUT}}$. A thermal-electricalluminous-chromatic model, including five submodels, is derived from RGB LED luminaire, where the four submodels, i.e., $E_{P}, E_{T}, Y_{P}$, and $Y_{T}$, were modeled as temperature-dependent or electrical-dependent due to low quantum efficiency, spectral shift, and current droop by the junction temperature rise. Compared to [15], a converter and a compensator, which are designed as compact linear models and instead of a lookup table, are introduced in the TFF\&FFB structure. The advantage is that the lighting system can operate at wide color range and would not restrict at specific CCT conditions. In contrast, the disadvantage is that the compensator must be remodeled by the four compact linear models in each control period due to the temperaturedependent and electrical-dependent properties. A diagonal PI 
controller designed by decentralized control approach is shown concise and effective. The diagonal effect is because the photon emission is dominated by the electrical power of the corresponding color LEDs, and the thermal interaction is minor in contrast to the optical effect.

\section{CONCLUSION}

This paper has proposed a novel FFB\&TFF structure for an RGB LED lighting control system, in which a converter was applied for command conversion and a compensator was used to compensate the temperature variation and to adjust the signal mismatch between the converter outputs and initial responses of RGB LEDs. The RGB LED luminaire was modeled and experimentally identified as a thermal-electrical-luminous-chromatic model, including five submodels, where T-L, T-C, and E-C models were treated as functions of electrical power and E-L model as a function of junction temperature in order to simplify the modeling calculation of the compensator. The converter was obtained from the experimental results of T-C model identification. From the point of view of decentralized control approach, the control system designed in the three SISO loops with PI diagonal controller could guarantee system stability and performance. Finally, the designed controller was implemented for experimental verification. The experimental results of both transient and steady-state experiments showed low color drift and low total luminous intensity droop. Compared to previous work [15], the novel FFB\&TFF control structure can operate at a wide color range and would not restrict at specific CCT conditions. The proposed control system were shown to be effective.

\section{REFERENCES}

[1] E. F. Schubert and J. K. Kim, "Solid-state light sources getting smart," Science, vol. 308, no. 5726, pp. 1274-1278, May 2005.

[2] Y. Gu, N. Narendran, T. Dong, and H. Wu, "Spectral and luminous efficacy change of high-power LEDs under different dimming methods," in Proc. SPIE, 2003, vol. 6337, p. 63370J. Available: http://proceedings. spiedigitallibrary.org/proceeding.aspx?articleid $=1291526$

[3] M. Dyble, N. Narendran, A. Bierman, and T. Klein, "Impact of dimming white LEDs: Chromaticity shifts due to different dimming methods," in Proc. SPIE, 2005, vol. 5941, pp. 291-299.

[4] S. Muthu, F. J. Schuurmans, and M. D. Pashley, "Red, green, and blue LEDs for white light illumination," IEEE Trans. J. Sel. Top. Quantum Electron., vol. 8, no. 2, pp. 333-338, Mar./Apr. 2002.

[5] C. Hoelen, J. Ansems, P. Deurenberg, T. Treurniet, E. van Lier, O. Chao, V. Mercier, G. Calon, K. van Os, G. Lijten, and J. Sondag-Huethorst, "Multi-chip color variable LED spot modules," in Proc. SPIE, 2005, vol. 5941, p. 59410A. Available: http://proceedings.spiedigitallibrary. org/proceeding. aspx? articleid $=1330744$.

[6] P. Deurenberg, C. Hoelen, J. van Meurs, and J. Ansems, "Achieving color point stability in RGB multi-chip LED modules using various color control loops," in Proc. SPIE, 2005, vol. 5941, pp. 63-74.

[7] C. Hoelen, J. Ansems, P. Deurenberg, W. van Duijneveldt, M. Peeters, G. Steenbruggen, T. Treurniet, A. Valster, and J. W. ter Weeme, "Color tunable LED spot lighting," in Proc. SPIE, 2006, vol. 6337, p. 63370Q. Available: http://proceedings.spiedigitallibrary.org/ proceeding.aspx? articleid $=1291531$.

[8] S. Muthu, F. J. Schuurmans, and M. D. Pashley, "Red, green, and blue LED based white light generation: Issues and control," in Proc. Ind. Appl. Conf., 2002, pp. 327-333.

[9] S. Muthu and J. Gaines, "Red, green, and blue LED-based white light source: Implementation challenges and control design," in Proc. Ind. Appl. Conf., 2003, pp. 515-522.

[10] B. Ackermann, V. Schulz, C. Martiny, A. Hilgers, and X. Zhu, "Control of LEDs," in Proc. IEEE IAS'06, Oct., pp. 2608-2615.

[11] K.-C. Lee, S.-H. Moon, B. Berkeley, and S.-S. Kim, "Optical feedback system with integrated color sensor on LCD," Sens. Actuator A, Phys., vol. 130-131, pp. 214-219, 2006
[12] S.-Y. Lee, J.-W. Kwon, H.-S. Kim, M.-S. Choi, and K.-S. Byun, "New design and application of high efficiency LED driving system for RGBLED backlight in LCD display," in Proc. IEEE PESC'06, Jun., pp. 1-5.

[13] K. Lim, J. C. Lee, G. Panotopulos, and R. Helbing, "Illumination and color management in solid state lighting," in Proc. IEEE IAS'06, Oct., pp. 2616-2620.

[14] S. Robinson and I. Ashdown, "Polychromatic optical feedback control, stability, and dimming," in Proc. SPIE, 2006, vol. 6337, p. 633714.

[15] F.-C. Wang, C.-W. Tang, and B.-J. Huang, "Multivariable robust control for a red-green-blue LED lighting system," IEEE Trans. Power Electron., vol. 25, no. 2, pp. 417-428, Feb. 2010.

[16] E. F. Schubert, Light Emitting Diodes. Cambridge,, U.K.: Cambridge University Press, 2003.

[17] J. Senawiratne, A. Chatterjee, T. Detchprohm, W. Zhao, Y. Li, M. Zhu, Y. Xia, X. Li, J. Plawsky, and C. Wetzel, "Junction temperature, spectral shift, and efficiency in GaInN-based blue and green light emitting diodes," Thin Solid Films, vol. 518, no. 6, pp. 1732-1736, Jan. 2010.

[18] M.-H. Kim, M. F. Schubert, Q. Dai, J. K. Kim, E. F. Schubert, J. Piprek, and Y. Park, "Origin of efficiency droop in GaN-based light-emitting diodes," Appl. Phys. Lett., vol. 91, no. 18, p. 183507, 2007. Available: http://apl.aip.org/resource/1/applab/v91/i18/p183507_s1.

[19] D. Saguatti, L. Bidinelli, G. Verzellesi, M. Meneghini, G. Meneghesso, E. Zanoni, R. Butendeich, and B. Hahn, "Investigation of efficiencydroop mechanisms in multi-quantum-well InGaN/GaN blue light-emitting diodes," IEEE Trans. Electron Devices, vol. 59, no. 5, pp. 1402-1409, May 2012.

[20] B.-J. Huang, C.-W. Tang, and M.-S. Wu, "System dynamics model of highpower LED luminaire," Appl. Therm. Eng., vol. 29, no. 4, pp. 609-616, 2009

[21] B.-J. Huang and C.-W. Tang, "Thermal-electrical-luminous model of multi-chip polychromatic LED luminaire," Appl. Therm. Eng., vol. 29, no. 16, pp. 3366-3373, 2009.

[22] S. S. Hui and Y. X. Qin, "A general photo-electro-thermal theory for light emitting diode (LED) systems," IEEE Trans. Power Electron., vol. 24, no. 8, pp. 1967-1976, Aug. 2009.

[23] G. Wyszecki and W. S. Stiles, Color Science, Concepts and Methods, Quantitative Data and Formulae, 2nd ed. New York, NY, USA: Wiley, 1982

[24] A. R. Robertson, "Computation of correlated color temperature and distribution temperature," J. Opt. Soc. Amer., vol. 58, pp. 1528-1535, 1968.

[25] Everlight Electronic Co. RGGB high power LED - 4W datasheet EHPB02. (2008). [Online]. Available: http://www.everlight.com/

[26] Hamamatsu Photonics. Si photodiode S1133 datasheet. (2008). [Online]. Available: http://hamamatsu.com/

[27] M. Doshi and R. Zane, "Control of solid state lamps using a multiphase pulse width modulation technique," IEEE. Trans. Power Electron., vol. 25, no. 7, pp. 1894-1904, Jul. 2010.

[28] D. L. Blackburn, "Temperature measurements of semiconductor devices-A review," Proc. 20th Ann. IEEE Semicond. Therm. Meas. Manag. Symp., pp. 70-80, Mar. 2004.

[29] T.-P. Sun and C.-H. Wang, "Specially designed driver circuits to stabilize LED light output without a photodetector,"," IEEE Trans. Power Electron., vol. 27, no. 3, pp. 1598-1607, 2012.

[30] X. Tao, H. Chen, S. N. Li, and S. Y. Hui, "A new noncontact method for the prediction of both internal thermal resistance and junction temperature of white light-emitting diodes," IEEE Trans. Power Electron., vol. 27, no. 4, pp. 2184-2192, Apr. 2012.

[31] S. Skogestad and I. Postlethwaite, Multivariable Feedback Control, Analysis and Design, 2nd ed. New York, NY, USA: Wiley, 2000.

[32] P. Albertos and S. Antonio, Multivariable Control Systems an Engineering Approach. New York, NY, USA: Springer-Verlag, 2003.

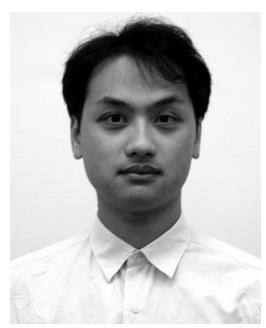

Chun-Wen Tang was born in Taipei, Taiwan, in 1976. He received the B.S. degree in mechanical engineering from the National Taiwan University of Science and Technology, Taipei, in 1990, and the M.Sc. and $\mathrm{Ph} . \mathrm{D}$. degrees from the Control Group, Mechanical Engineering Department, National Taiwan University, Taipei, in 2000 and 2009, respectively.

$\mathrm{He}$ is currently a Project Manager at the Industrial Technology Research Institute, Hsinchu, Taiwan. His current research interests include light-emitting diode lighting and control, independent solar photovoltaic (PV) system, PV/T system, solar microgrid technology system, zero-energy technology, greenhouse engineering, and emotional design. 


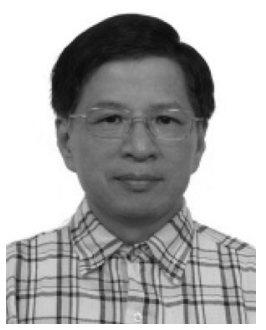

Bin-Juine Huang received the Master's degrees in mechanical and chemical engineering from Case Western Reserve University, USA and the doctorate degree from Odessa State Academy of Refrigeration, Odessa, Ukraine.

$\mathrm{He}$ is currently a Professor in the Department of Mechanical Engineering, National Taiwan University, Taipei, Taiwan, where he is the Director of the Solar Energy Research Center (SERC), which is supported by the Global Research Partnership (GRP) Award of King Abdullah University of Science and Technology (KAUST). He was involved in researching a broad array of fields, including energy systems (solar photovoltaic (PV), geothermal, ocean thermal, wind, boiler, and waste heat), cooling technology (absorption, ejector, desiccant, cryocoolers, and thermoelectric), solid-state lighting (light-emitting diode), and control technology. His current research include bridging the gap between academia and industry. He has developed more than 30 products with industry. He is the author or coauthor of more than 200 academic papers and 150 technical reports. He holds more than 60 worldwide patents.

Prof. Huang was a recipient of the 1972 Outstanding Youth of the Year Award, the 1991 National Outstanding Engineering Professor Award, the 1995 Academician of Academy of Sciences of Technological Cybernetics of Ukraine Award, the 1996 Academician of International Academy of Refrigeration, Ukraine Branch Award, the 1996 Tong-Yuan Science and Technology Award, the 2000 Outstanding Researcher Award of the National Science Council, the 2005 Science and Technology Award of China-Tech Foundation, and the 2005 Solar and New Energy Contribution Award of the Solar and New Energy Society of Taiwan.

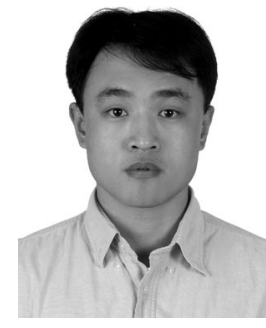

Shang-Ping Ying was born in Taiwan in 1968. He received the Ph.D. degree in photonics (fabrication and characterization of II-VI semiconductor microstructure doped glass) from the National Chiao Tung University, Hsinchu, Taiwan, in 1999.

He was the Opto-Electronics and Systems Laboratories, Industrial Technology Research Institute of Taiwan, Hsinchu, where he was involved in the epitaxy of GaN, illumination and color management of red-green-blue light-emitting diodes (LEDs), light source technology in photodynamic therapy (PDT), optical simulation, and optical system design in the fields of solid state lighting. He is currently an Assistant Professor in the Department of Opto-Electronic System Engineering, Minghsin University of Science and Technology, Hsinchu. His current research interests include high-power LED packaging technology, chip-on board LED modules, phosphor characteristic for white LED, and accurate and reliable optical-electrical measurements of high-power LEDs. 